\title{
Vom Text zur Übung. Kollokationen in Lesetexten und ihre Widerspiegelung in Übungen (am Beispiel ausgewählter \\ DaF-Lehrwerke)
}

Joanna Targońska (Olsztyn)

\begin{abstract}
Collocations are an important part of lexis. Their use is often indispensable and they cannot be replaced by any other lexical means. Unfortunately, they pose a big problem for learners of a foreign language because they are not conspicuous and the learners are not aware of their conventionality and fixed character. It is then important to draw the learner's attention to that part of lexis, for example by placing collocations in the vocabulary practice. This analysis attempts to investigate to what degree the lexical practice following specific texts in a coursebook incorporates the collocations that have been used in those texts. The analysis focuses on the coursebooks for German as a foreign language for learners at B1+ level. The objective of the analysis is to check if the students' attention is directed to collocations in the texts themselves and then if the collocations are part of the vocabulary practice. The research concerns the degree to which the collocations in the texts become part of the vocabulary practice (quantitative analysis) and their quality (qualitative analysis).
\end{abstract}

\section{$1 \quad$ Einleitung}

Kollokationen stellen einen wichtigen und nicht zu unterschätzenden Teil des Wortschatzes jeder (zumindest europäischen) Sprache dar. Sie bilden einen Bestandteil sowohl der gesprochenen als auch der geschriebenen Sprache und drücken lexikalisch wichtige Sachverhalte aus, wobei sie sich dort oft nicht oder nur schwer umschreiben bzw. durch andere lexikalische Mittel ersetzen lassen. Muttersprachler ${ }^{1}$ sind sich der Existenz dieses Sprachphänomens nicht bewusst, dennoch erwerben sie Kollokationen aufgrund ihrer Aussetzung gegenüber dem kollokationsreichen Input, dem sie Kollokationen als Ganzes unbewusst auf eine natürliche Weise entnehmen. Wie aus empirischen Studien hervorgeht, werden Kollokationen, die sich leicht aus dem Kontext erschließen lassen, im Leseprozess nicht automatisch erworben (cf. Marton 1977; Gabryś-Biskup 1990; Bahns/Sibilis 1992). Deshalb sind ihre Vermittlung (seitens der Lehrkraft bzw. anhand des von ihr eingesetzten Lehrwerks) und ihr Erwerb (seitens der DaFLernenden) im Fremdsprachen- und darunter im DaF-Unterricht(sprozess) enorm wichtig.

\footnotetext{
${ }^{1}$ Im vorliegenden Artikel wird bewusst nicht gegendert und die maskulinen Formen schließen sowohl Frauen als auch Männer ein.
} 
Das Lernen von Kollokationen erfordert eine bewusste Aufmerksamkeitsfokussierung auf die Form der Kollokation, insbesondere auf den Kollokator, der bei der Bedeutungserschließung „unauffällig“ zu sein vermag. Aus diesem Grund stellt die explizite Auseinandersetzung mit Kollokationen in DaF-Lehrwerken eine Notwendigkeit dar. Diese kann unterschiedlich realisiert werden (cf. Targońska 2015c). Eine der Möglichkeiten der bewussten Lenkung der Aufmerksamkeit der DaF-Lernenden auf die Bedeutung und die Form der Kollokationen bzw. ihre syntaktischen Besonderheiten ist, Kollokationen zum Gegenstand lexikalischer Übungen zu machen. Eine andere Möglichkeit besteht darin, Kollokationen mit der Text- und der Wortschatzarbeit zu verbinden, wobei die zuerst genannte Möglichkeit den ersten didaktischen Schritt darstellen könnte, gefolgt von Wortschatzübungen².

Das Ziel des Beitrags ist die Untersuchung des Zusammenhangs der Text- und Wortschatzarbeit in ausgewählten DaF-Lehrwerken. Dabei soll analysiert werden, inwieweit die in Lesetexten vorkommenden Kollokationen daraus exzerpiert und in die lexikalischen Übungen transferiert werden und dort den Übungsgegenstand darstellen und ob Wortschatz- sowie andere Übungen die Kollokabilität der deutschen Sprache widerspiegeln. Der Beitrag zielt auf die Beantwortung der Frage ab, ob die DaF-Lernenden auf eine unterschiedliche Art und Weise auf bestimmte Kollokationen explizit aufmerksam gemacht werden, ob die in Texten auftretenden Kollokationen in lexikalischen Übungen aufgegriffen werden oder doch keinen Gegenstand des lexikalischen Übens darstellen. Darüber hinaus soll die Qualität der Übungen, die die Kollokationen berücksichtigen, analysiert werden.

Der vorliegende Beitrag besteht aus folgenden Teilen: Im ersten Teil (Kap. 2) wird das Verständnis des Kollokationsbegriffs in dieser Arbeit dargelegt. Der nächste Abschnitt (Kap. 3) widmet sich Kollokationen aus der Sicht der Spracherwerbsforschung. Hier werden Gründe dafür angegeben, warum dieses Sprachphänomen den Fremdsprachenlernenden Probleme bereitet. Weiterhin wird auf die Relevanz der textbasierten Arbeit an Kollokationen eingegangen (Kap. 4). Den umfangreichsten Teil bildet die Beschreibung einer empirischen Studie. Im Kap. 5 werden die Ergebnisse der Lehrwerkanalyse dargestellt. Abschließende Bemerkungen und aus den Ergebnissen der Lehrwerkanalyse resultierende didaktische Implikationen bilden das Ende des Beitrags.

\section{$2 \quad$ Zum Kollokationsbegriff}

Da der Kollokationsbegriff unterschiedlich aufgefasst werden kann (im Allgemeinen unterscheidet man zwar zwischen der weiteren, d. h. syntaktischorientierten und der engeren, d. h. semantisch- bzw. bedeutungsorientierten Auffassung des Kollokationsbegriffs ${ }^{3}$ ) und sein Ver-

\footnotetext{
2 Diese Vorgehensweise würde auch dem phraseologischen Dreischritt (cf. Kühn 1992) entsprechen und somit auch einen der ersten Schritte der heutigen Phraseodidaktik darstellen. Immer öfter wird nämlich die Meinung vertreten, dass der erste Schritt der Phraseo- und somit auch der Kollokationsdidaktik die Entwicklung des Phrasem- bzw. Kollokationsbewusstseins darstellen sollte (cf. dazu Holzinger 1993: 14; Laskowski 2004, 2010; Reder 2015), dank dem Fremdsprachenlernende Kollokationen im sprachlichen Input (z. B. in Lesetexten) einfacher wahrnehmen und erkennen können.

${ }^{3}$ Diese grobe Einteilung in der Auffassung des Kollokationsbegriffs kann jedoch den fälschlichen Eindruck erwecken, dass es nur zwei unterschiedliche Deutungsmöglichkeiten des Kollokationsbegriffs gibt. Diese sind je nach dem Autor recht unterschiedlich. Auch innerhalb der engen Auffassung gibt es Meinungsunterschiede, z. B. hinsichtlich der Aufnahme bzw. Nicht-Aufnahme von Funktionsverbgefügen bzw. von Streckformen der Verben sowie der lexikalischen Solidaritäten von Coseriu (1967) usw. in die Gruppe der Kollokationen.
} 
ständnis variieren kann, ist es in jeder Arbeit unabdingbar, sein terminologisches Verständnis, das den Gegenstand einer bestimmten Untersuchung darstellt, darzulegen.

Im Folgenden stütze ich mich zum einen auf die engere Auffassung des Terminus bei Hausmann $(1984,1985,2003)$, der nicht nur der bekannteste Vertreter der bedeutungs- bzw. semantikorientierten Auffassung des Kollokationsbegriffs ist, sondern der auch als erster auf die hierarchische, d. h. Basis-Kollokator-Struktur der Kollokationen hingewiesen hat. Zum anderen basiert das Verständnis dieses Terminus auf der fremdsprachendidaktischen Deutung dieses Terminus von Reder (2006) und Targońska (2015a), die zwischen der engeren und der weiteren zu positionieren ist. Dies bedeutet, dass im Folgenden Hausmanns Auffassung des Kollokationsbegriffs um eine kleine Gruppe der festen konventionellen Wortverbindungen erweitert wird, was unten näher dargestellt wird.

Kollokationen werden im Folgenden als eine Gruppe syntagmatischer Wortverbindungen verstanden, die sich durch Festigkeit, Assoziativität bzw. Gerichtetheit (cf. Gładysz 2003), Idiosynkrasie und Arbitrarität (cf. Irsula Peña 1994: 96; Forkl 2010: 62; Konecny 2010a: 155-157), Affinität (cf. Hausmann 1984), Direktionalität bzw. Evozierbarkeit (cf. Häcki Buhofer 2011: 522; Roth 2014: 27) auszeichnen. Als eine phraseologische Kombination (cf. Hausmann 2003) 4 stellen sie eine Untergruppe der Phraseologismen (diese im weiteren Sinne) dar, weil sie sich durch keine Idiomatizität auszeichnen, die für Phraseologismen im engeren Sinne charakteristisch ist. Dies bedeutet jedoch nicht, dass sie eine Randerscheinung in der Phraseologie darstellen. Aus der Sicht der sprachlichen Kommunikation und der fremdsprachlichen Wortschatzkompetenz scheinen sie viel wichtiger als idiomatische Phraseologismen zu sein, deren Kenntnis eher für die Sprachrezeption notwendig ist ${ }^{5}$. Viele Kommunikationssituationen erfordern jedoch den produktiven Gebrauch von Kollokationen, die als eine Ganzheit aus dem mentalen Lexikon abgerufen werden (müssen). Diese sollten, neben den Routineformeln, von den niedrigsten Stufen der Sprachbeherrschung an produktiv beherrscht werden.

$\mathrm{Zu}$ Kollokationen werden in dieser Arbeit solche festen konventionellen Syntagmen gerechnet, in denen die Zusammenstellung der Glieder nicht nur fest, sondern auch konventionell, d. h. in der Sprachnorm begründet ist. Im Laufe der Zeit scheinen sich manche Wortverbindungen verfestigt zu haben, weil der Gebrauch eines Wortes (eines Kollokators) bevorzugt wurde (cf. Szulc 1975: 32). Somit haben wir im Fall der Kollokationen mit lexikalischen Restriktionen in der Zusammenstellung der Glieder in diesem konventionellen Syntagma zu tun, worauf Caro Cedillo (2004: 88) folgendermaßen hinweist: „Konstitutives Merkmal der

\footnotetext{
${ }^{4}$ Laut Hausmann (2003: 84) ist eine Kollokation dann phraseologisch, ,,wenn sie in der vorliegenden Form in der Sprache (der Sprache als Norm, nicht nur der Sprache als System) üblich ist und vor anderen, theoretisch möglichen, bevorzugt wird“"

${ }^{5}$ Die Änderung in der Phraseodidaktik betrifft gerade die (Nicht)Notwendigkeit der produktiven Beherrschung von Phraseologismen. Während noch in der Anfangsphase der Phraseodidaktik die Anwendungsphase den letzten phraseodidaktischen Schritt darstellte (cf. Kühn 1992) und die Relevanz der produktiven Beherrschung sich auf die ganze Phraseologie bezog, wird seit einigen Jahren die Meinung vertreten, dass nicht alle Phraseologismen von Fremdsprachenlernenden produktiv zu beherrschen sind und nicht alle von den niedrigsten Niveaustufen an, worauf Lüger (1997: 101) schon vor 20 Jahren hingewiesen hat. Auch Ettinger (2007: 869; 2011: 239; 2013: 16-18) ändert im Laufe der Zeit seine Meinung in dieser Hinsicht und setzt sich für die produktive Beherrschung von nicht allen Phraseologismen ein. Produktiv sollten nach ihm insbesondere Routineformeln schon von der niedrigsten Stufe der Sprachbeherrschung an beherrscht werden.
} 
Kollokation ist in erster Linie die lexikalische Restriktion, die auf die konventionalisierte, normbedingte, idiosynkratische Art der Wortverbindung zurückzuführen ist“". Lexikalische Restriktionen äußern sich in der eingeschränkten lexikalischen Kombinierbarkeit der Kollokationsglieder, d.h. in der Tatsache, dass die semantisch autonome Kollokationsbasis aus einer Gruppe möglicher Kollokationspartner einen bestimmten Kollokator selegiert, obwohl semantisch gesehen auch andere Wörter theoretisch als Kollokatoren auftreten könnten. Hartenstein (1991: 67) bezeichnet diese Besonderheit der Kollokationen als „beschränkte lexikalische (oder) lexematische Kombinierbarkeit“. Darunter versteht er ,solche ausdrucksseitigen (oder signifikantbezogenen) Restriktionen seiner [des Lexems] Verbindbarkeit mit anderen Lexemen [...], die sich nicht aus der Unverträglichkeit der betreffenden Wortbedeutungen untereinander ergeben, sondern daraus, daß der Vorkommensbereich des Lexems im Hinblick auf andere, mit ihm semantisch kompatible Lexeme eingeschränkt ist" (ibd.). Auch in der bedeutungsorientierten Auffassung des Kollokationsbegriffs ist die Gruppe der Kollokationen nicht einheitlich. Hier kann man sogar drei Untergruppen unterscheiden, wobei für die jeweilige Untergruppe unterschiedliche Eigenschaften der Kollokationen (nicht immer alle der oben erwähnten) charakteristisch sind.

Im Folgenden wird in Anlehnung an Hausmann (1984) eine hierarchische Struktur der Kollokationen angenommen. Diese bestehen demnach aus einer Kollokationsbasis, die semantisch autonom ist und oft in der primären Bedeutung auftritt und einem Kollokator, der von der Kollokationsbasis semantisch abhängig ist. Zwischen den Kollokationsgliedern, die sich gegenseitig semantisch beeinflussen, besteht eine spezifische Beziehung. Die semantische Abhängigkeit des Kollokators äußert sich darin, dass seine Bedeutung erst in Bezug auf die Kollokationsbasis erklärt werden kann, was Hausmann (2003: 83) folgendermaßen ausführt: „Die Basis ist ein Wort, das ohne Kontext definiert, übersetzt und gelernt werden kann. Der Kollokator ist ein Wort, das nicht ohne Kontext übersetzt werden kann. [...] Erst im Kontext wird der Kollokator definierbar, übersetzbar und lernbar"6. Das Verb halten beispielsweise kann unterschiedliche Bedeutungen annehmen, je nachdem, mit welchem Substantiv bzw. welcher Kollokationsbasis es in einem Syntagma oder einer Kollokation auftritt. Dieses Verb hat unterschiedliche Bedeutungen z. B. in folgenden Kollokationen: einen Vortrag halten, das Versprechen/das Wort halten, Abstand halten, sich an die Regeln halten usw. Hier ist die Bedeutung des Kollokators nur durch die Bezugnahme auf die Kollokationsbasis zu erklären, weil er von der Kollokationsbasis semantisch abhängig ist. In den Syntagmen ein Buch halten oder ein Kind in den Armen halten, die freie Wortverbindungen darstellen, ist die Bedeutung des Verbs halten gleich, unabhängig davon, mit was für einem Substantiv dieses Verb gebraucht wird. Dies gilt auch für das Verb halten für, dessen Rektion schon auf seine Bedeutung hinweist. Hier verändert jedes dazu gebrauchte Substantiv als Akkusativobjekt die Bedeutung des Verbs nicht.

Der Kollokator tritt zwar oft in einer übertragenen Bedeutung bzw. in der zweiten Lesart auf, aber diese Besonderheit betrifft nicht alle Kollokationen. In manchen Kollokationen, wie z. B. Zähne putzen, steht der Kollokator in seiner „,wörtlichen“ Bedeutung, kann aber durch kein

\footnotetext{
${ }^{6}$ Dieses Merkmal bezieht sich auf die zweite und dritte Gruppe der von Reder (2006) postulierten Kollokationsarten.
}

ISSN 1615-3014 
synonymes Verb ersetzt werden ${ }^{7}$. Diese Kollokationen stellen die zweite Untergruppe der Kollokationen dar, für die die konventionsbedingte Kookkurrenz der Bestandteile charakteristisch ist.

In die Gruppe der Kollokationen wurden im Folgenden auch solche Wortverbindungen aufgenommen, deren Festigkeit daraus resultiert, dass ein Kollokationsglied ein eingeschränktes semantisches Potenzial hat (cf. Reder 2006: 79). Es tritt nur in einer bestimmten Wortverbindung auf (z. B. Nase rümpfen, Hass schüren, Bier brauen, Hunde bellen). Dies führt dazu, dass der Gebrauch eines Kollokationsgliedes bei Hörern und Sprechern den Gebrauch eines weiteren konkreten Gliedes erwarten lässt. Dies ist die dritte Untergruppe der Kollokationen.

Somit schieße ich mich im Folgenden auch Reders (2006: 77-79) Auffassung der Kollokationen an, laut der Kollokationsglieder folgende Determinationsbeziehungen aufweisen können:

- das eingeschränkte Beziehungspotenzial eines Bestandteiles ${ }^{8}$,

- eine semantische Abhängigkeitsbeziehung der Bestandteile,

- $\quad$ eine konventionsbedingte Kookkurrenz der Bestandteile.

Dabei ist zu betonen, dass nicht jede Kollokation all diese Determinationsbeziehungen aufweist. Die erste Eigenschaft bezieht sich auf solche Wörter, ,deren Bedeutung so spezifisch ist, dass sie nur mit einigen wenigen anderen Wörtern vorkommen“ (Reder 2006: 79). „Die Eigenschaft des stark eingeschränkten Beziehungspotenzials kann also der jeweilige Kollokator in einer niedrigen Zahl der möglichen Partner realisieren“ (ibd.: 80). Zwar wird die erste Gruppe der Kollokationen, die auch den lexikalischen Solidaritäten ${ }^{9}$ entspricht, oft nicht in die enge, bedeutungsorientierte Auffassung des Kollokationsbegriffs aufgenommen, diese findet aber ihren Platz in der Gruppe der Kollokationen nach dem fremdsprachendidaktischen Verständnis dieses Begriffs (wie bei Reder 2006; Burger 2007; Hausmann 2007; Konecny 2010a; Targońska 2015a).

In der in diesem Beitrag angenommenen fremdsprachendidaktischen Auffassung des Kollokationsbegriffs (cf. Targońska 2015a) werden kognitive Prozesse beim Fremdsprachenlernen berücksichtigt. Sowohl beim produktiven Gebrauch als auch in der Sprachrezeption gehen die Denkprozesse von der Kollokationsbasis aus. Erstens wird die Bedeutung des Kollokators nur durch die Bezugnahme auf die Kollokationsbasis erschlossen. Zweitens soll der Kollokator in der Sprachproduktion erst durch die Bezugnahme auf die Kollokationsbasis gewählt werden. Der Sprachbenutzer soll dabei darüber reflektieren, ob der Kollokator der jeweiligen Kollokationsbasis (unter Bezugnahme auf die sprachliche Norm) entspricht. Dabei wird Abstand sowohl von der korpuslinguistischen Auffassung des Kollokationsbegriffs genommen, in der

\footnotetext{
${ }^{7}$ In diesem Fall haben wir es auch mit einer konventionellen Wortverbindung zu tun, denn im Englischen lautet dieses Syntagma brush one's teeth. Der englische Kollokator entspricht also dem deutschen Verb bürsten (dieses Lexem existiert jedoch im Deutschen in dem Kompositum Zahnbürste). Auf Polnisch lautet dieses Syntagma myć zęby (also *,Zähne waschen“) oder szczotkować zęby (*,Zähne bürsten“), wobei die erste Kollokation gemeinsprachlich und die zweite eher fachsprachlich ist.

${ }^{8}$ Diese Gruppe der festen Wortverbindungen findet auch ihren Platz in der späteren Auffassung des Kollokationsbegriffs von Hausmann (2007: 218). Hier verweist er auf lexikalische Solidaritäten, die unter den Kollokationsbegriff subsumiert werden können, folgendermaßen: „Lexikalische Solidaritäten des Typs der Hund bellt sind ein Sonderfall, insofern der Kollokator bellen ohne Kontext definiert werden kann, aber nicht ohne Nennung des Basiswortes bei der Definition“.

${ }^{9}$ Konecny (2010a) nimmt auch lexikalische Solidaritäten in die Gruppe der Kollokationen auf.
} 
statistisch signifikante Kookkurrenzen als Kollokationen gedeutet werden ${ }^{10}$, als auch davon, was einmal als Kollokation, ein anderes Mal als „Konstruktion“11 bezeichnet wird. Nicht bei allen Konstruktionen (zu denen z. B. auch Zwillingsformeln und grammatische Valenzen gehören) (cf. auch Siepmann 2004) lässt sich die Kollokationsbasis-Kollokator-Struktur unterscheiden ${ }^{12}$. Hier kann man nicht zwischen dem bedeutungstragenden und dem bedeutungsspezifizierenden Element differenzieren. Zum anderen nimmt die Verfasserin hier Abstand von dem rein linguistischen Verständnis der Kollokationen. Der Autorin des vorliegenden Beitrags ist es bewusst, dass Linguisten (z. B. Wotjak/Heine 2005: 147) bzw. Korpuslinguisten (z. B. Wallner 2014) Funktionsverbgefüge (weiter FVG) aus der Gruppe der Kollokationen ausschließen, jedoch finden diese ihren Platz in der fremdsprachendidaktischen Auffassung der Kollokationen, weil in ihnen, wie in vielen anderen Kollokationen, das Verb dem Substantiv konventionell zugeordnet wird und dieses seine Bedeutung erst in der Verbindung mit einem bestimmten Substantiv (z. B. der Kollokationsbasis) bekommt. Die Aufnahme von FVG in die Gruppe der Kollokationen ist nicht neu, weil dies viele Kollokationsforscher tun. So sieht Burger (2007: 54) ihren Platz unter den Kollokationen, wobei er diese als ,die regulärste Untergruppe der Substantiv-Verb-Kollokationen“ bezeichnet. Für Krenn (2004: 2) stellen FVG „spezielle Verb-Objekt-Kollokationen“ und für Irsula Peña (1996: 75) „SubstantivVerb-Kollokationen mit prädikativer Funktion“ dar. Hausmann (2007: 218) stellt Folgendes fest: „Funktionsverbgefüge (Eid leisten) und Vergleichsformen (dumm wie Bohnenstroh) haben Kollokationsstruktur (Basis: Eid und dumm)“. Auch Lüger (2004), Ludewig (2005), Reder (2006) und Konecny (2010a) subsumieren FVG unter ihre Auffassungen des Kollokationsbegriffs.

Andererseits wird u. a. Siepmann (2004), Hollós (2010) und Wallner (2014) zugestimmt, dass Kollokationen nicht nur zweigliedrige Wortverbindungen darstellen und dass diese oft durch ein zusätzliches (oft gerade obligatorisches Element) ergänzt werden. Dies bedeutet jedoch nicht, dass diese keine binäre Struktur aufweisen. Kollokationen können sich nämlich zu Tripel-Konstruktionen (cf. Hausmann 2004: 316) verbinden, die entweder aus zwei Kollokationen gebildet oder durch ein zusätzliches Element ergänzt bzw. vervollständigt werden können. In der binären Auffassung der Kollokationen, die im Folgenden angenommen wird, muss sich jedoch die Kollokationsbasis-Kollokator-Struktur unterscheiden lassen, was z. B. für Zwillingsformeln nicht gilt.

Viele Wissenschaftler, die sich mit dem fremdsprachendidaktisch bzw. lexikographisch orientierten Kollokationsbegriff beschäftigen, betonen, dass die Grenzen zwischen Kollokationen und ihren benachbarten Sprachphänomenen, darunter insbesondere die Grenze zu freien Wortverbindungen, fließend sind (cf. Konecny 2010a). Diese Meinung vertritt auch die Autorin des vorliegenden Beitrags, die sich dessen bewusst ist, dass manche Kollokationen von

\footnotetext{
${ }^{10}$ Viele Autoren, die sich des korpuslinguistischen Verständnisses des Kollokationsbegriffs bedienen, sprechen auch von „usuellen Wortverbindungen“ (cf. Katochvilová 2004).

${ }^{11}$ Unter Konstruktionen werden im Folgenden Teile „wiederholter Rede“ verstanden, die sowohl lexikalische und grammatische Kollokationen als auch andere Routineformeln umfassen. Beispiele für Konstruktionen sind z. B. gern gemacht, nicht zu danken, auf Schritt und Tritt, sich freuen auf. Siehe dazu die Fußnote 12.

12 Siepmann (2004: 110) schlägt z. B. als andere Arten der Kollokationen folgende Verbindungen vor: Verb + Infinitiv, Verb + Präposition, Adjektiv + Infinitiv. Schon der Gebrauch des Wortes Infinitiv weist darauf hin, dass es sich um eine grammatische und keine lexikalische Konstruktion handelt. Im Falle einer Wortverbindung des Typs Verb + Präposition geht es um die Rektion des Verbs.
} 
einigen Wissenschaftlern für freie Wortverbindungen, von anderen dagegen für Kollokationen gehalten werden. In solchen Fällen, in denen schwer zu entscheiden ist, ob es sich schon um eine Kollokation oder noch um eine freie Wortverbindung handelt, wird die Konventionalität und einzelsprachliche Besonderheit dieser Wortverbindungen unter die Lupe genommen, wobei bei der Feststellung des konventionellen Charakters der Kollokationen eine kontrastive Betrachtung des Deutschen mit dem Polnischen vorgenommen wird ${ }^{13}$.

\section{$3 \quad$ Kollokationen als Lernproblem}

Kollokationen stellen ein Lernproblem dar, worauf viele Kollokationsforscher hinweisen (cf. Bahns 1997: 65-73; Nesselhauf 2003; Reder 2006; Konecny 2010a). Auf Probleme der Fremdsprachenlernenden, die aus der Verbindbarkeit der Wörter resultieren, hat schon vor über 50 Jahren Leonhardi aufmerksam gemacht. Er stellte Folgendes fest:

Die Schwierigkeit bei der Erlernung einer fremden Sprache liegt weder in der Aneignung isolierter Wörter noch in den Formen und Fügungen der fremden Sprache selbst, sie liegt in der Versuchung, Kombinationen der Muttersprache in die fremde Sprache zu übernehmen.

(1964: 21-22)

Szulc $(1971,1975)$ hat jahrelang auf Kollokationen, d. h. konventionelle bzw. konventionalisierte Syntagmen als problematischen Bereich des Erwerbs von fremdsprachigem Wortschatz hingewiesen (cf. Targońska 2015d). Einen ähnlichen Hinweis auf Kollokationen als interferenzträchtigen Bereich finden wir bei Konecny:

Für Fremdsprachenlerner sind Kollokationen [...] besonders tückisch: Auch wenn sie die entsprechende Fremdsprache [...] gut beherrschen, sind sie gerade im Bereich der Kollokationen immer wieder der Gefahr von Interferenzfehlern ausgesetzt, weil sie normalerweise unbewusst dazu tendieren, die Wortverbindungen wörtlich von der Mutter- in die Fremdsprache zu übertragen und damit gegen die lexikalischen Kollokationsrestriktionen der Zielsprache zu verstoBen

(2010b: 79)

Kollokationen stellen ein Lernproblem, d.h. eine Ursache für Interferenzfehler aus einem bestimmten Grund dar, nämlich der Unbewusstheit dieses Sprachphänomens aufseiten der Fremdsprachenlernenden, die sich der konventionellen Verbindbarkeit der Lexeme in diesem konventionellen, normbedingten Syntagma in vielen Sprachen nicht bewusst sind. Diese Verbindbarkeit unterliegt bestimmten Beschränkungen bzw. lexikalischen (und bei manchen Kollokationen auch grammatischen) Restriktionen ${ }^{14}$. Die Unkenntnis dieser Tatsache führt dazu, dass Fremdsprachenlernende einerseits kreativ Lexeme in einem Syntagma zusammenstellen. Andererseits stützen sie sich dabei auf die muttersprachliche Norm und verbinden Lexeme der jeweiligen Fremdsprache anhand dieser L1-Norm. Sie übertragen somit die „Denkweise“ der Erstsprache oder einer besser beherrschten Fremdsprache auf die Zielsprache (cf. Szulc 1971, 1984). Die Unbewusstheit dieses Sprachphänomens seitens der DaF-Lernenden ist auf zwei Faktoren zurückzuführen. Auf der einen Seite ist dieses Sprachphänomen vielen (wenn nicht den meisten DaF-Lehrkräften) nicht geläufig. Auf der anderen Seite scheinen dieses Sprach-

\footnotetext{
${ }^{13}$ Oft erlaubt erst die Gegenüberstellung zweier Sprachen die Feststellung der Konventionalität dieser Wortverbindungen.

${ }^{14}$ Den Hinweis auf grammatische Restriktionen bzw. syntaktische Präferenzen mancher Kollokationen finden wir z. B. bei Siepmann (2002) und Wallner (2014).
}

ISSN 1615-3014 
phänomen und seine Relevanz im DaF-Lernprozess in DaF-Lehrwerken nicht explizit dargestellt zu werden (cf. Targońska 2015b). Probleme mit inkorrektem Gebrauch von Kollokationen resultieren ferner daraus, dass korrekt rezipierte Kollokationen nicht produktiv beherrscht werden. Dies liegt daran, dass viele Fremdsprachenlernende (insbesondere jene mit einem nicht gut entwickelten Sprachbewusstsein, die über die Struktur der Sprachen nicht reflektieren) dem Kollokator keine Aufmerksamkeit schenken.

\section{Kollokationen in Lehrwerken - Warum die textbasierte Arbeit an Kollokatio- nen?}

Aus dem oben Dargelegten lässt sich die Schlussfolgerung ziehen, dass DaF-Lernende für Kollokationen und insbesondere für den Kollokator als deren fehlerträchtigsten Teil sensibilisiert werden müssen. Auch ihr Kollokationsbewusstsein sollte gefördert bzw. gestärkt werden. Bei der Realisierung dieser beiden Ziele könnte das Lehrwerk behilflich sein. Dies ist damit zu begründen, dass nicht-muttersprachliche DaF-Lehrkräfte nicht alle deutschen Kollokationen kennen und selbst Fehler in diesem Bereich machen.

Zur Förderung der Kollokationskompetenz ist es wichtig, über das Lernen von Kollokationslisten hinauszugehen (cf. Siepmann 2004: 113), wenn man die Fremdsprachenlernenden auf lebenslanges Lernen von Kollokationen vorbereiten möchte. Das Lehrwerk steuert das Unterrichtsgeschehen oft am stärksten und somit kann es eine Hilfe bzw. einen Ausgangspunkt zur Förderung der Kollokationskompetenz darstellen. Zwar kann dieses Sprachphänomen in den Lehrwerken angehängten Glossaren berücksichtigt oder in manchen Wortschatzübungen implizit behandelt werden, aber auch textbasierte Arbeit an Kollokationen ${ }^{15}$ ist aus zwei Gründen wichtig. Während der Textarbeit, bei der von Fremdsprachenlernenden nicht nur der Inhalt, sondern auch die sprachliche Form fokussiert werden kann, kann kollokationsentdeckendes Lesen betrieben werden. Dieses stellt die erste Etappe der Kollokationsdidaktik (vgl. dazu die Fußnote 2) dar (Bahns 1993; Gładysz 2003) und dient der Förderung des Kollokationsbewusstseins. Laut Hausmann (1984: 406) geht es bei der Textarbeit um die Bewusstmachung der in einem gerade besprochenen Text auftretenden Kollokationen und um die Angabe von deren Äquivalenten in der jeweiligen Muttersprache. Wichtig ist jedoch die Verbindung der Textarbeit mit den auf sie folgenden Übungen, denn erkannte und bewusst exzerpierte Kollokationen müssen geübt und danach angewendet werden (zwei aufeinander folgende Phasen der Arbeit an Kollokationen). In der Kollokationsdidaktik, die nach Reder (2006: 203) in drei Schritten erfolgen sollte: (1) Entdecken der Kollokationen als Einheiten, (2) Einüben der Kollokationen als Einheiten und (3) Anwenden der Kollokationen als Einheiten, bildet das Erkennen der Kollokationen den ersten wichtigen Schritt. Dieser soll gerade anhand der formfokussierenden Textarbeit erfolgen. Diese verfolgt eigentlich zwei Ziele. Erstens sollen dabei Kollokationen entdeckt werden, zweitens soll dabei Kollokationsbewusstsein gefördert werden (cf. Bahns 1993: 33-35). Wie das kollokationale Lehren und Lernen erfolgen kann, stellt sich Siepmann (2014: 13) folgendermaßen vor: Wichtiger als das Lernen von Kollokationen

\footnotetext{
15 Hausmann (1984: 406) nennt zwei Typen der Kollokationsarbeit: erstens Kollokationsarbeit am Text, zweitens mit dem zweisprachigen Wörterbuch. Auf die Berücksichtigung der Kollokationen in der Wortschatzdidaktik verwies Hausmann (1976: 56) schon vor 40 Jahren folgendermaßen: „Das Sammeln von Kollokationen aus motivierenden L2-Texten muss auf allen Stufen des Wortschatzlernens in den Vordergrund treten“.
}

ISSN 1615-3014 
in Form von Wortlisten ist laut ihm die Suche nach Kollokationen in gesprochener und geschriebener Sprache. Nachdem das globale Verständnis eines Textes geprüft worden ist, können sich die Lernenden beim zweiten Lesen bzw. Hören auf die Ermittlung der Kollokatoren $\mathrm{zu}$ den angegebenen Basen konzentrieren. Auch bestimmte Übungen (Verbindung der Arbeit am Inhalt mit der an sprachlichen Formen) können die Lernenden dazu anregen und anleiten. Diese Arbeit an Kollokationen soll zum Erwerb der Kollokationskompetenz als einer wichtigen Subkompetenz der Wortschatzkompetenz führen.

\section{Kollokationen in Lesetexten und ihre Widerspiegelung in lexikalischen Übungen - empirische Untersuchung}

Diese Studie stellt eine Ergänzung der Studien von Targońska (2015b) dar, in denen Kollokationen in DaF-Lehrwerken der Niveaustufen A1 und A2 (cf. Targońska 2015b) sowie in Vokabeltaschenbüchern (cf. Targońska 2016) auf die Vermittlung von Kollokationen in Vokabellisten und in Beispielsätzen untersucht wurden. In Targońska (2015b) wurden DaFLehrwerke in Bezug auf die explizite und implizite Behandlung von Kollokationen analysiert, wobei der Analyse nur Wortschatzübungen und Glossare unterzogen wurden. In dieser Studie werden Lehrwerke unter einem anderen Aspekt analysiert. Im folgenden Teil des Beitrags wird der Zusammenhang zwischen Lesetexten und Wortschatzübungen untersucht und die textbasierte Kollokationsarbeit unter die Lupe genommen.

\subsection{Forschungsziel und Forschungsfragen}

Die an dieser Stelle beschriebene Untersuchung ist eine Lehrwerkanalyse, wobei ihr gezielt Lesetexte und Übungen (insbesondere lexikalische) unterzogen wurden. Das Ziel der vorliegenden Studie ist es zu erfassen, ob und inwieweit Lehrwerkautoren die in Lesetexten vorkommenden Kollokationen exzerpieren und zum Übungsgegenstand machen und somit kollokationsentdeckendes Lesen fördern, bzw. kollokationales Wortschatzlernen anregen. Im Folgenden wird der Zusammenhang zwischen Texten und Wortschatzübungen analysiert.

Die vorliegende Studie ist auf die Beantwortung folgender Forschungsfragen ausgerichtet:

1. Werden die in Lesetexten auftretenden Kollokationen in Wortschatzübungen aufgegriffen und zum Übungsgegenstand gemacht?

2. Werden die Lernenden durch die lexikalischen Übungen zum formorientierten Lesen der Texte ermuntert bzw. angeregt?

3. Können mittels der (lexikalischen) Übungen kollokationsentdeckendes Lesen und Kollokationsbewusstsein gefördert werden?

4. Welcher Anteil der in Lesetexten auftretenden Kollokationen wird zum Übungsgegenstand gemacht?

5. In welche Art der Übungen werden diese in Lesetexten vorkommenden Kollokationen integriert?

6. Werden „neue“ Kollokationen in lexikalischen Übungen eingeführt und eingeübt?

7. Fokussieren diese Übungen, in denen Kollokationen auftreten, ihre Form oder ihre Bedeutung?

Die Analyse der Lehrwerke ist sowohl quantitativ als auch qualitativ ausgerichtet, denn von Interesse ist nicht nur die Erfassung der Anzahl von Kollokationen, die aus den Texten exzer- 
piert und zum Gegenstand der Wortschatzübungen gemacht wird, sondern auch die Art und Weise der didaktischen Umsetzung des Postulats vom kollokationalen Lernen.

\subsection{Untersuchungsgegenstand und Forschungsvorgehensweise}

Für die Analyse wurden zwei Lehrwerkpakete ausgewählt, nämlich das Lehrwerk Aspekte B1+ (Koithan et al. 2007a, 2007b) und Sicher B1+ (Perlmann-Balme/Schwalb 2012; OrthChambah/Perlmann-Balme/Schwalb 2012), wobei bei jedem dieser Lehrwerke sowohl das Kurs- als auch das Arbeitsbuch analysiert wurden. Der Entscheidung für Lehrwerke auf dieser Niveaustufe lag zum einen der Gedanke zugrunde, dass darin schon viele längere, kohärente Texte aus verschiedenen Bereichen zu finden sind. Zum anderen geht aus empirischen Studien hervor, dass Kollokationsfehler die wichtigste Fehlerart bei fortgeschrittenen Fremdsprachen-, darunter auch DaF-Lernenden, sind. Diese produzieren viele inkorrekte Kollokationen, weil sie in ihren Sprachkenntnissen schon fortgeschritten sind, weswegen sie mit lexikalischen Einheiten kreativ umgehen, und zwar auch dort, wo dies nicht möglich ist. Ihnen scheint jedoch das Kollokationsphänomen nicht bewusst zu sein, weil Lehrkräfte sie auf Kollokationen bzw. Kollokabilität der Sprachen nicht aufmerksam machen (cf. Targońska 2014: 139).

Gewählt wurden gerade solche Lehrwerke, die eine breite Palette an Wortschatzübungen aufweisen. Weiterhin sollten es internationale, weltweit eingesetzte Lehrwerke von verschiedenen Verlagen sein. Wichtig war auch hier die Möglichkeit der Gegenüberstellung eines älteren Lehrwerks (Aspekte B1+, Langenscheidt 2008) einem neueren (Sicher B1+, Hueber 2012). Nicht analysiert wurden im Folgenden zusätzliche Materialien zu den Lehrwerken in Form von Vokabeltrainings bzw. Arbeitsblättern, die von Verlagen ins Internet gestellt werden bzw. die in Lehrerhandreichungen zu finden sind. Dies ist damit zu erklären, dass nicht alle Lernenden von dem Angebot der Online-Materialien Gebrauch machen. Bei der Analyse sollten solche Lehr- und Lernmaterialien untersucht werden, mit denen alle mit diesem Lehrwerk arbeitenden DaF-Lernenden konfrontiert werden ${ }^{16}$.

Den Untersuchungsgegenstand stellt eine Untergruppe der Kollokationen dar, nämlich Substantiv-Verb-Kollokationen, die auch als verbale Kollokationen bezeichnet werden. Ausgewählt wurde diese Gruppe der Kollokationen, weil sie die häufigste Art der Kollokationen darstellen und von DaF-Lernenden öfter gebraucht werden als andere Kollokationen.

Der Hauptteil des Titels des vorliegenden Beitrags lautet „Vom Text zur Übung“, weswegen bei der Analyse der Lehrwerke von der Untersuchung der Lesetexte ausgegangen wurde. Diese wurden auf das Vorkommen von Substantiv-Verb-Kollokationen des Typs Substantiv (als Subjekt) + Verb und Substantiv (als Objekt bzw. Präpositionalobjekt) + Verb hin analysiert $^{17}$. Anschließend wurden Wortschatzübungen auf die Behandlung der gleichen Kollokationen wie in den Lesetexten hin untersucht. Als Ausgangspunkt wurde angenommen, dass

\footnotetext{
${ }^{16}$ Der Autorin des vorliegenden Beitrags ist es bewusst, dass in Lehrwerken nicht alles der Reihe nach gemacht bzw. ,abgehakt“ wird und Lehrkräfte manche Seiten, Texte bzw. Übungen überspringen. Aber wenn etwas in einem Lehrwerk steht, kann der Lernende auch selbstständig manche Übungen und Aufgaben machen. Im Folgenden wird also das Angebot analysiert und nicht dessen Gebrauch bzw. Umsetzung.

${ }^{17} \mathrm{Im}$ Lehrwerk Apekte B1+ wurden Texte aus dem Kurs- und Arbeitsbuch analysiert, ausgenommen der Abschnitte der literarischen Texte, sowie Texte im landeskundlichen Teil „Porträt“, zu dem keine (lexikalischen) Übungen erstellt wurden.
} 
Kollokationen dann den Gegenstand des sprachlichen Übens darstellen, wenn in der jeweiligen Übung ihre Form bzw. ihre Bedeutung fokussiert wird ${ }^{18}$. Problematisch war die Entscheidung, ob Übungen zum mündlichen bzw. schriftlichen Ausdruck, in denen Kollokationen implizit behandelt werden, auch in die Analyse mit einbezogen werden sollten. Da die DaFLernenden in diesen Übungen zum Gebrauch der angeführten Kollokationen angeregt werden ${ }^{19}$, wurden diese Übungen auch in der Studie berücksichtigt.

Im zweiten Schritt wurden alle Wortschatzübungen auf das Vorkommen und Üben von anderen, in Lesetexten nicht vorkommenden verbalen Kollokationen hin analysiert. Bei der Analyse der Lehrwerke wurde auf der einen Seite untersucht, welcher Anteil der in Lesetexten auftretenden Kollokationen in die Wortschatzübungen transferiert wird, d. h. den DaF-Lernenden bewusst gemacht und weiterhin eingeübt wird. Auf der anderen Seite wurde analysiert, ob in lexikalischen Übungen auch Kollokationen den Übungsgegenstand darstellen und welche neuen Kollokationen erst einfach in Wortschatzübungen eingeführt und geübt werden. Die Analyse der beiden Lehrwerke erfolgt separat, wobei am Ende die Thematisierung der Kollokationen in beiden Lehrwerken gegenübergestellt wird.

\subsection{Kollokationen in Aspekte B1}

Der Darstellung der Analyseergebnisse soll eine kurze Charakteristik des Lehrwerks vorangestellt werden, denn diese könnte eine Erklärung für die Ergebnisse der Untersuchung liefern. Aspekte $B 1+$ besteht aus 10 Kapiteln, in denen sehr viele Lesetexte zu finden sind. Die Anzahl der Lesetexte variiert je nach Kapitel und schwankt zwischen fünf und sieben, wobei ihre Länge unterschiedlich ist. Zwei bzw. drei der Texte in jedem Kapitel sind relativ lang, die anderen eher kurz. Die Anzahl und die Länge der Texte lassen erwarten, dass darin relativ viele Substantiv-Verb-Kollokationen vorkommen. Auch die Anzahl der Übungen ist groß. Diese sind sowohl im Lehrbuch als auch im Arbeitsbuch zu finden.

Die Analyse von Lesetexten in diesem Lehrwerk zeigt, dass Substantiv-Verb-Kollokationen zwar in Lesetexten vorkommen, aber ihre Anzahl variiert zum einen je nach dem Kapitel, zum anderen je nach dem Text. Die meisten Kollokationen kommen in Kapiteln 1, 3, 5 und 10 vor. Die wenigsten Kollokationen treten in Lesetexten in den Kapiteln 4 und 8 auf, was der Tabelle $1 \mathrm{zu}$ entnehmen ist.

\begin{tabular}{|l|c|c|c|}
\hline Kapitel & $\begin{array}{l}\text { Anzahl der in Lesetexten } \\
\text { vorkommenden Kolloka- } \\
\text { tionen }\end{array}$ & $\begin{array}{l}\text { Anzahl dieser Kollokationen (aus } \\
\text { Lesetexten), die in lexikalischen } \\
\text { Übungen berücksichtigt wurden }\end{array}$ & $\begin{array}{l}\text { Anzahl der erst in Wort- } \\
\text { schatzübungen eingeführ- } \\
\text { ten Kollokationen }\end{array}$ \\
\hline Kap. 1 & 17 & 1 & 0 \\
\hline Kap.2 & 10 & 5 & 9 \\
\hline Kap.3 & 9 & 2 & 15 \\
\hline
\end{tabular}

\footnotetext{
18 Die Autorin dieses Beitrags ist sich dessen bewusst, dass Form und Bedeutung zwei eng miteinander verbundene Elemente der lexikalischen Einheiten sind, weil ihre Bedeutung auch aus ihrer Form resultiert. Da im Falle der Kollokationen die Erschließung ihrer Bedeutung oft kein Problem für die Fremdsprachenlernenden darstellt, ist gerade der Hinweis auf die Form der Kollokation (darunter die Art des konventionell zur Kollokationsbasis zugeordneten Kollokators oder grammatische Restriktionen, die für viele Kollokationen gelten) von großer Bedeutung.

${ }^{19}$ Diese Übungen gehören zur letzten, d. h. zur Anwendungsphase, in der gerade kennengelernte Kollokationen in der Sprachkommunikation gebraucht werden (müssen).
} 


\begin{tabular}{|l|c|c|c|}
\hline Kap. 4 & 6 & 0 & 5 \\
\hline Kap. 5 & 13 & 0 & 3 \\
\hline Kap. 6 & 6 & 1 & 9 \\
\hline Kap. 7 & 8 & 0 & 0 \\
\hline Kap. 8 & 4 & 0 & 1 \\
\hline Kap. 9 & 10 & 0 & 0 \\
\hline Kap. 10 & 12 & 0 & $\mathbf{4 3}$ \\
\hline Insgesamt & $\mathbf{9 5}$ & $\mathbf{9}$ & 1 \\
\hline
\end{tabular}

Tabelle 1: Kollokationen in Lesetexten und in lexikalischen Übungen in Aspekte B+

An dieser Stelle sollen einige in Lesetexten vorkommende Kollokationen exemplarisch dargestellt werden. Dabei konzentrieren wir uns zuerst auf Kollokationen aus den Kapiteln 1 und 2.

\begin{tabular}{|c|c|c|c|c|}
\hline Lp. & Kollokationen in Lesetexten & $\begin{array}{l}\text { Kapitel, } \\
\text { LB/AB Seite }\end{array}$ & $\begin{array}{l}\text { Berück- } \\
\text { sichtigt in lex. } \\
\text { Übungen }^{20}\end{array}$ & $\begin{array}{l}\text { Berücksichtigung bzw. Ein- } \\
\text { führung der Kollokationen } \\
\text { in Wortschatzübungen }\end{array}$ \\
\hline 1. & eine Ausbildung als ... machen & 1, LB. 9 & & \\
\hline 2. & einen Traum erfüllen & 1, LB. 10 & & \\
\hline 3. & eine CD aufnehmen & 1, LB. 10 & & \\
\hline 4. & $\begin{array}{l}\text { seinen Lebensunterhalt verdie- } \\
\text { nen }\end{array}$ & 1, LB. 10 & & \\
\hline 5. & seinen Traum verwirklichen & $\begin{array}{l}\text { 1, LB. } 10,1, \mathrm{AB}, \\
8,2, \mathrm{~KB}, 26\end{array}$ & & \\
\hline 6. & seinen Traum ausleben & $1, \mathrm{LB}, 10$ & & \\
\hline 7. & einen Traum umsetzen & $1, \mathrm{AB}, 8$ & & \\
\hline 8. & der Brand bricht aus & $1, \mathrm{AB}, 12$ & & \\
\hline 9. & Drillinge bekommen & $1, \mathrm{~KB}, 17$ & & \\
\hline 10. & in den Sinn kommen & $1, \mathrm{~KB}, 17$ & & \\
\hline 11. & seine Wünsche zurückstellen & $1, \mathrm{~KB}, 17$ & & \\
\hline 12. & sich eine Auszeit gönnen & $1, \mathrm{~KB}, 17$ & $\mathrm{X}$ & $\begin{array}{l}\mathrm{KB}, 3 \mathrm{~b} / 17 \text { eine bedeutungs- } \\
\text { fokussierende Zuordnungsü- } \\
\text { bung }\end{array}$ \\
\hline 13. & eine Versicherung abschließen & $1, \mathrm{AB}, 15$ & & \\
\hline 14. & eine Wohnung mieten & $1, \mathrm{AB}, 15$ & $\mathrm{X}$ & $\begin{array}{l}\text { AB 1/24. Die Zuordnung der } \\
\text { zum Substantiv die Wohnung } \\
\text { passenden Verben }\end{array}$ \\
\hline 15. & das Glück hüten & $1, \mathrm{AB}, 15$ & & \\
\hline 16. & den Hobbys nachgehen & $1, \mathrm{AB}, 15$ & & \\
\hline 17. & & 2, AB Üb.3b/19 & & Hausordnung einhalten \\
\hline 18. & & 2, AB Üb. 3b/19 & & Wohngemeinschaft gründen \\
\hline
\end{tabular}

${ }^{20}$ Mit einem X wird markiert, welche in Lesetexten vorkommenden Kollokationen in lexikalische Übungen transferiert wurden und dort den Gegenstand des lexikalischen Übens darstellen.

ISSN 1615-3014 


\begin{tabular}{|c|c|c|c|c|}
\hline 19. & & $\begin{array}{l}\text { 2, AB Üb. 3b/19, } \\
\text { 2, AB Üb. 1/18 }\end{array}$ & & Mietvertrag unterschreiben \\
\hline 20. & & 2, AB Üb. 1/24 & & die Wohnung einrichten \\
\hline 21. & & $2, \mathrm{AB}$ Üb. $1 / 24$ & & die Wohnung besichtigen \\
\hline 22. & & 2, AB Üb. $1 / 24$ & & $\begin{array}{l}\text { eine Wohnung untervermie- } \\
\text { ten }\end{array}$ \\
\hline 23. & & $2, \mathrm{AB}$ Üb. $1 / 24$ & & eine Wohnung streichen \\
\hline 24. & & 2, AB Üb. $1 / 26$ & & Verantwortung übernehmen \\
\hline 25. & & 2, AB Üb. 4a/28 & & eine Einweihungsparty geben \\
\hline 26. & einen Beruf erlernen & $2, \mathrm{AB}, 23$ & & \\
\hline 27. & die Regeln einhalten & $2, \mathrm{AB}, 23$ & & \\
\hline 28. & ins Berufsleben eintreten & $2, \mathrm{~KB}, 32$ & & \\
\hline 29. & Wehr- oder Ersatzdienst leisten & $2, \mathrm{~KB}, 32$ & & \\
\hline 30. & das Ziel erreichen & $2, \mathrm{~KB}, 32$ & & \\
\hline 31. & Kosten abschätzen & $2, \mathrm{AB}, 27$ & & \\
\hline 32. & eine Kaution hinterlegen & $2, \mathrm{AB}, 27$ & $\mathrm{X}$ & $\begin{array}{l}\text { AB 3b/19 Zuordnung der } \\
\text { Verben zu den Substantiven }\end{array}$ \\
\hline 33. & Anschluss finden & $2, \mathrm{AB}, 27$ & & \\
\hline
\end{tabular}

Tabelle 2: Kollokationen in Kapitel 1 und Kapitel 2 (Aspekte B1+)

Wie aus der Tabelle 2 hervorgeht, kommen in den ersten beiden Kapiteln Kollokationen in Lesetexten sowohl im Lehr- als auch im Arbeitsbuch vor, wobei in manchen nur einzelne Kollokationen auftreten und in anderen mehrere (z. B. im Text „Gelebte Träume“, KB, S. 10 und im Text „Man muss etwas tun für sein Glück“, KB, S. 17 konnten jeweils 5 Kollokationen ausfindig gemacht werden). Von den fast 30 Kollokationen, die in Lesetexten der beiden Kapitel ermittelt wurden, wurden nur drei, nämlich sich eine Auszeit gönnen, eine Wohnung mieten und eine Kaution hinterlegen aus dem Lesetext exzerpiert und zum Übungsgegenstand gemacht. Die erste Kollokation wurde in eine Zuordnungsübung integriert (vgl. Graphik 1), in der die Aufmerksamkeit der Lernenden nur auf ihre Bedeutung, jedoch nicht auf ihre Form, gelenkt wurde. Hier wurde diese Kollokation in eine Übung mit anderen Phrasemen (darunter Idiomen sowie mit Wortbildungsprodukten [z. B. unvermittelbar sein] integriert).

b Lesen Sie den Text. Unterstreichen Sie die Ausdrücke 1-5 im Text und ordnen Sie die passenden Erklärungen a-e zu.

$1 \_$jemandem / sich eine Auszeit gönnen a keine Zusage bekommen können

2 _ jemandem unter die Arme greifen $\quad b$ jemandem behilflich sein, helfen

3 _ etwas fällt nicht vom Himmel c gleichzeitig, auf einmal

4 _ unvermittelbar sein d etwas ist nicht ohne Mühe zu erreichen

5 _ auf einen Streich e sich Freizeit nehmen, Pause machen

Graphik 1: Berücksichtigung der Kollokation sich eine Auszeit gönnen in einer Übung (Aspekte B1+, KB 3b/17) 
Die Kollokation eine Wohnung mieten wurde in eine Übung ( $\mathrm{AB} 1 / 24)$ eingebettet, in der zum Substantiv Wohnung nicht passende Verben zu erkennen sind. Die Kollokation eine Kaution hinterlegen $(\mathrm{AB}$ 1b/19) wird auch in eine Zuordnungsübung (dem Substantiv soll ein passendes Verb zugeordnet werden) eingebettet, wobei sie zuerst in einer Übung erscheint (Anfang des Kapitels) und dann (am Ende des Kapitels) in einem Text vorkommt.

Einer separaten Analyse bedarf das Auftreten und die Behandlung der Kollokationen in den Kapiteln 3 und 6 (vgl. Tabelle 3 und 4). Das dritte Kapitel unterscheidet sich von den übrigen in zweierlei Hinsicht. Zum einen werden hier die meisten Kollokationen aus Lesetexten in Wortschatzübungen transferiert. Es handelt sich dabei um folgende Kollokationen: Medikamente absetzen, Glückshormone ausschütten und Schmerzen vertreiben, wobei die letzte Kollokation den Gegenstand von zwei Übungen darstellt. Interessanterweise wurden diese Kollokationen in Übungen zu den grammatischen Phänomenen Präfixverben und Gebrauch des Perfekts integriert, wobei hier auch der neue Wortschatz eingeübt werden sollte. In diesen Übungen wurde die Aufmerksamkeit der DaF-Lernenden auf den Kollokator, insbesondere auf seine grammatische Form gelenkt. Erwähnenswert ist eine andere Vorgehensweise in der Übung (AB 2/32). Darin wird die Kollokation Schmerzen vertreiben geübt, wobei die DaFLernenden in einer Zusammenfassung des Lesetextes die Kollokationsbasis zum Kollokator vertreiben ergänzen müssen. Dieses Kapitel unterscheidet sich von anderen auch dadurch, dass hier viele Kollokationen gerade erst in Wortschatzübungen eingeführt und gleichzeitig geübt wurden. Es handelt sich dabei um die Kollokationen von 13 bis 19 (vgl. Tabelle 3).

\begin{tabular}{|c|c|c|c|c|}
\hline Lp. & $\begin{array}{l}\text { Kollokationen in Lese- } \\
\text { texten }\end{array}$ & $\begin{array}{l}\text { Kapitel, } \\
\text { LB/AB } \\
\text { Seite }\end{array}$ & $\begin{array}{l}\text { Berück- } \\
\text { sichtigt } \\
\text { in lex. } \\
\text { Übungen }\end{array}$ & $\begin{array}{l}\text { Berücksichtigung bzw. Einführung der } \\
\text { Kollokationen in Wortschatzübungen }\end{array}$ \\
\hline 1. & Muskeln anspannen & $3, \mathrm{~KB}, 42$ & & \\
\hline 2. & Muskeln entspannen & $3, \mathrm{~KB}, 42$ & & \\
\hline 3. & $\begin{array}{l}\text { Glückshormone aus- } \\
\text { schütten }\end{array}$ & $3, \mathrm{~KB}, 42$ & $\mathrm{X}$ & $\begin{array}{l}\text { Üb. 5/33 (Wortbildungsübung. Präfixe } \\
\text { müssen ergänzt werden) }\end{array}$ \\
\hline 4. & $\begin{array}{l}\text { den Anstoß für etwas } \\
\text { geben }\end{array}$ & $3, \mathrm{~KB}, 42$ & & \\
\hline 5. & Medikamente absetzen & $3, \mathrm{~KB}, 42$ & $\mathrm{X}$ & $\begin{array}{l}\text { Üb 6/ } 33 \text { (Ergänzungsübung - Sätze } \\
\text { müssen mit einem entsprechenden Verb } \\
\text { in der Partizip-II-Form ergänzt werden) }\end{array}$ \\
\hline 6. & & $\mathrm{AB}, \ddot{\mathrm{Ub}} .3 / 31$ & & den Blutdruck messen \\
\hline 7. & & AB Üb. $3 / 31$ & & eine Diagnose stellen \\
\hline 8. & & AB Üb. 3/31 & & ein Rezept ausstellen \\
\hline 9. & & AB Üb. 3/31 & & einen Zahn ziehen \\
\hline 10. & Schmerzen vertreiben & $3, \mathrm{~KB}, 42$ & $\mathrm{X}$ & $\begin{array}{l}\text { Üb. } 2 / 32 \text { - Kollokationsbasis muss zum } \\
\text { Kollokator vertreiben ergänzt werden. } \\
\text { Üb. } 5 / 33 \text { - Wortbildungsübung. Präfixe } \\
\text { müssen ergänzt werden. }\end{array}$ \\
\hline 11. & $\begin{array}{l}\text { jemanden zum Lachen } \\
\text { bringen }\end{array}$ & $3, \mathrm{~KB}, 42$ & & \\
\hline 12. & $\begin{array}{l}\text { eine Therapie anwen- } \\
\text { den }\end{array}$ & $3, \mathrm{~KB}, 42$ & $\mathrm{X}$ & Üb. 5/33 (Übung zu Präfixverben) \\
\hline
\end{tabular}




\begin{tabular}{|c|c|c|c|c|}
\hline 13. & & AB Üb. $6 / 33$ & & Tabletten einnehmen \\
\hline 14. & & AB Üb. 6/33 & & ein Medikament verschreiben \\
\hline 15. & & AB Üb. $7 / 33$ & & den Oberkörper frei machen \\
\hline 16. & & AB Üb. $7 / 33$ & & die Luft anhalten \\
\hline 17. & & AB Üb. $7 / 33$ & & die Salbe einmassieren \\
\hline 18. & & AB Üb. $7 / 33$ & & den Verband erneuern \\
\hline 19. & & AB Üb. $7 / 33$ & & die Tabletten auflösen \\
\hline 20. & Uhr verstellen & $3, \mathrm{~KB}, 48$ & & \\
\hline 21. & Saft auspressen & $3, \mathrm{AB}, 37$ & $\mathrm{X}$ & $\begin{array}{l}\text { Üb. AB1b/37 Phrasen zur Vorbereitung } \\
\text { einer Speise sollen herausgeschrieben } \\
\text { werden }\end{array}$ \\
\hline 22. & $\begin{array}{l}\text { etwas wird in den } \\
\text { Hintergrund gedrängt }\end{array}$ & $3, \mathrm{AB}, 35$ & & \\
\hline 23. & $\begin{array}{l}\text { jemanden aus dem } \\
\text { Schlaf reißen }\end{array}$ & $3, \mathrm{Ab}, 39$ & & \\
\hline
\end{tabular}

Tabelle 3: Kollokationen im Kapitel 3 (Aspekte B1+)

Das 3. Kapitel dieses Lehrwerks kann als ein Musterbeispiel für die Behandlung der Kollokationen betrachtet werden. Hier treten Kollokationen nicht nur in Lesetexten auf, sondern werden auch in Wortschatzübungen berücksichtigt. Die Vielfalt der Übungen, in denen die Form der Kollokationen im Fokus steht, ist groß. Die große Anzahl der Kollokationen im Kapitel 3 mag daraus resultieren, dass die Wortschatzbereiche „Gesundheit und Krankheit“ sowie „Essen" reich an Kollokationen sind.

Auf Kollokationen im Kap. 6 wird im Folgenden näher eingegangen, weil hier auf der einen Seite sehr viele Kollokationen erst in lexikalischen Übungen eingeführt werden (vgl. Tabelle 4). Auf der anderen Seite unterscheidet sich die Art dieser Übungen zu Kollokationen von übrigen in anderen Kapiteln.

\begin{tabular}{|c|c|c|c|c|}
\hline Lp. & $\begin{array}{l}\text { Kollokationen in Lesetex- } \\
\text { ten }\end{array}$ & $\begin{array}{l}\text { Kapitel, } \\
\text { LB/AB } \\
\text { Seite }\end{array}$ & $\begin{array}{l}\text { Berück- } \\
\text { sichtigt } \\
\text { in lex. } \\
\text { Übungen }\end{array}$ & $\begin{array}{l}\text { Berücksichtigung bzw. Einführung der Kol- } \\
\text { lokationen in Wortschatzübungen }\end{array}$ \\
\hline 1. & & AB Üb. 3/67 & & ein Telefonat führen \\
\hline 2. & & AB Üb. 3/67 & & ein Protokoll führen \\
\hline 3. & & AB Üb. 3/67 & & einen Vortrag halten \\
\hline 4. & & AB Üb. 3/67 & & $\begin{array}{l}\text { einen Vertrag unterschreiben } \\
\text { (Zuordnungsübung, einige Verben sollten } \\
\text { den angegebenen Substantiven zugeordnet } \\
\text { werden, darunter sind nicht nur feste, son- } \\
\text { dern auch viele freie Wortverbindungen) }\end{array}$ \\
\hline 5. & & $6, \mathrm{AB}, 68$ & & Geld verdienen, Üb. $1 \mathrm{a} / 68$ \\
\hline 6. & & $6, \mathrm{AB}, 68$ & & Überstunden machen, Üb. $1 \mathrm{a} / 68$ \\
\hline 7. & ein Unternehmen gründen & $6, \mathrm{AB}, 70$ & & \\
\hline 8. & Interesse wecken & $6, \mathrm{~KB}, 94$ & & \\
\hline
\end{tabular}




\begin{tabular}{|l|l|l|l|l|}
\hline 9. & Schafe und Kühe hüten & $6, \mathrm{~KB}, 97$ & $\mathrm{X}$ & $\begin{array}{l}\text { Üb. 1/96 KB Syntagmen werden Personen } \\
\text { zugeordnet. Ziel der Übung ist, das Erken- } \\
\text { nen von Bedeutung der Phrasen (darunter } \\
\text { der Kollokationen) und Hypothesenbildung. }\end{array}$ \\
\hline 10. & $\begin{array}{l}\text { Verantwortung überneh- } \\
\text { men }\end{array}$ & $6, \mathrm{~KB}, 97$ & $\mathrm{X}$ & $\begin{array}{l}\text { Anmerkung: Diese Kollokation wurde im } \\
\text { Kap. 2 in eine Übung (AB 1/26) eingebettet. }\end{array}$ \\
\hline 11. & sich auf den Weg machen & $6, \mathrm{~KB}, 97$ & & \\
\hline 12. & einen Schritt wagen & $6, \mathrm{~KB}, 97$ & & \\
\hline 13. & die Praxis eröffnen & $6, \mathrm{~KB}, 97$ & & eine Geschäftsreise machen \\
\hline 14. & & $6, \mathrm{~KB}, 96$ & & Patienten behandeln \\
\hline 15. & & $6, \mathrm{~KB}, 96$ & & \begin{tabular}{l} 
Vorträge halten \\
\hline 16.
\end{tabular} \\
\hline 17. & & $6, \mathrm{~KB}, 96$ & & $\begin{array}{l}\text { Termine einhalten } \\
\text { Eine kommunikativ ausgerichtete, inhalts- } \\
\text { orientierte, nicht formfokussierende Übung. } \\
\text { Ganze Phrasen sollen nur bestimmten Perso- } \\
\text { nen zugeordnet werden. }\end{array}$ \\
\hline
\end{tabular}

Tabelle 4: Kollokationen im Kapitel 6 (Aspekte B1+)

Erwähnenswert ist die Behandlung der Kollokationen im Modul 4 dieses Kapitels. Hier werden DaF-Lernende in einer Übungsform (Konfrontierung mit verschiedenen Phrasen bzw. Substantiv-Verb-Syntagmen) zur Hypothesenaufstellung angeregt (vgl. Graphik 2). Darunter sollten auch 5 Kollokationen (eine Geschäftsreise machen, Patienten behandeln, Vorträge halten, Kühe, Ziegen und Schafe hüten, Termine einhalten) bestimmten Fotos zugeordnet und in einem weiteren Schritt im mündlichen Ausdruck (Hypothesenbildung) gebraucht werden. Diese Übung ist als eine Vorbereitungsphrase zum Leseverstehen gedacht. Betonenswert ist jedoch die Tatsache, dass nicht alle der in dieser Übung eingeführten Kollokationen in dem Lesetext vorkommen. Nur die Kollokationen mit dem gleichen Kollokator Schafe/Kühe/Ziegen hüten kommen in dem Lesetext vor. Aber Kollokationen, die im Lesetext zu finden waren (Verantwortung übernehmen, sich auf den Weg machen, den Schritt wagen), wurden in der Übung zur Hypothesengenerierung nicht berücksichtigt, was bedauernswert ist. 




Graphik 2: Kollokationen als Redemittel zur Hypothesenbildung (Aspekte B1+, KB 1a/96)

In den Kapiteln 4, 5 und 7 bis 10 wurden keine in Lesetexten vorkommenden Kollokationen zum Übungsgegenstand gemacht. Dies bedeutet, dass in diesen Kapiteln den DaF-Lernenden keine Kollokationen explizit vermittelt bzw. bewusst gemacht werden. Daraus resultiert eine hohe Wahrscheinlichkeit, dass DaF-Lernende keine Kollokationen aus diesen Kapiteln internalisieren werden. Erwähnenswert ist die Tatsache, dass in einer einführenden Wortschatzübung zum Thema „Spielen“ Kollokationen eingeführt und geübt werden (AB Üb 1/44). Es handelt sich um folgende Kollokationen: eine Karte ziehen, zwei Felder vorrücken, eine Runde aussetzen.

In diesem Lehrwerk sind auch solche Kapitel zu finden, in denen viel mehr Kollokationen als in den übrigen vorkommen. Darin sind u. a. kollokationsreiche Texte enthalten, in denen mehr als fünf Kollokationen zu finden sind. Dies betrifft z. B. den folgenden Text aus dem Bereich der Medizin (vgl. Graphik 3). 


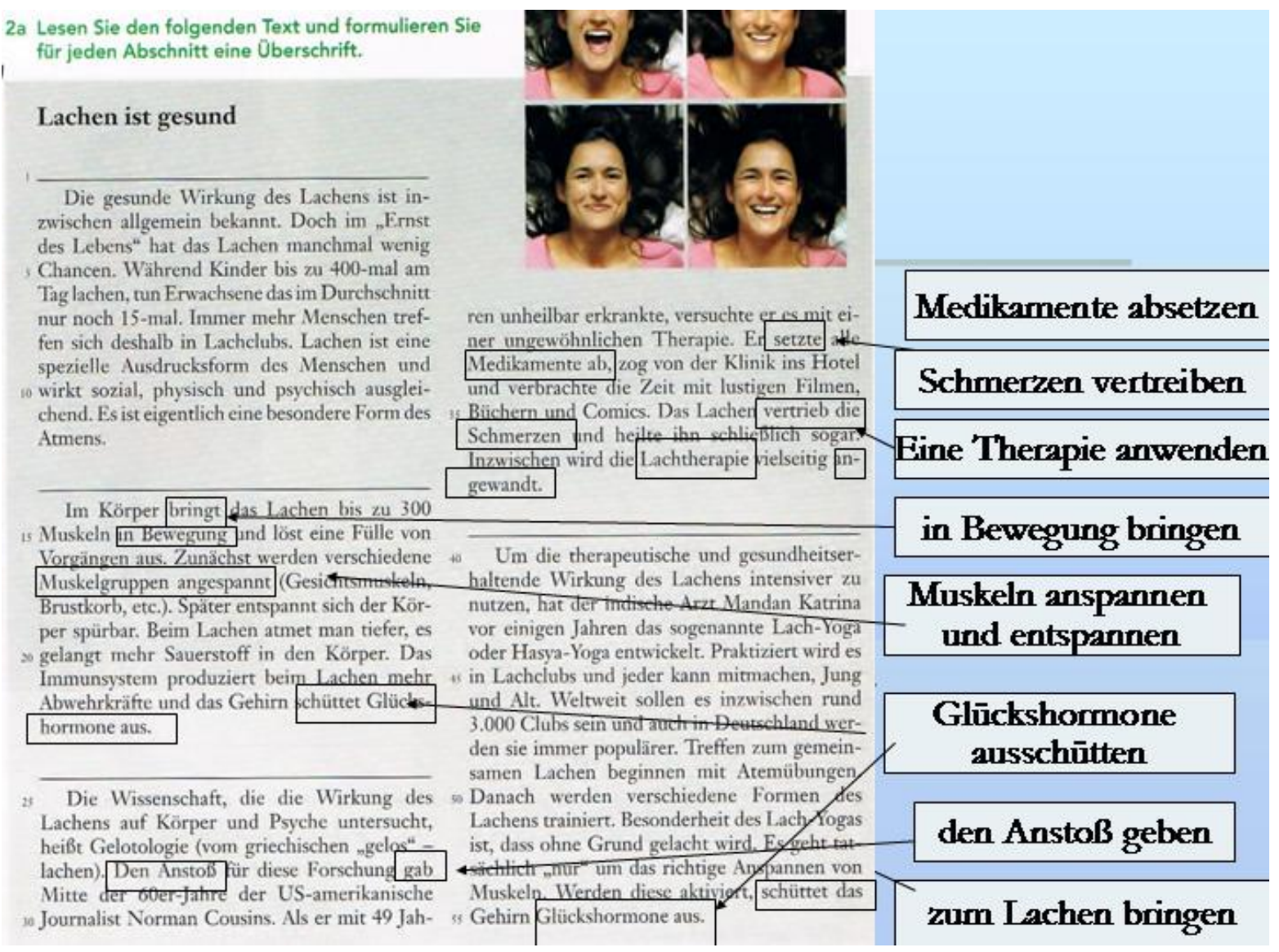

Graphik 3: Beispiel für einen kollokationsreichen Text (Aspekte B1+, KB S. 42)

Die Analyse des Lehrwerks hat ergeben, dass Kollokationen manchmal in lexikalischen Übungen auftreten, wobei sie dort keinen expliziten Übungsgegenstand darstellen. In diesen Fällen wurde die Möglichkeit, auf Kollokationen hinzuweisen und sie den DaF-Lernenden bewusst zu machen, nicht genutzt. Dies bezieht sich auf folgende Beispiele von 1 bis 3 (siehe unten) aus den Kapiteln 2 und 3. In Lückentexten bzw. Lückensätzen finden wir folgende Kollokationen (fett hervorgehoben von der Verfasserin):

1) Ich bin selbst schon mit 16 Jahren (ausgezogen/eingezogen/eingerichtet), weil ich eine Ausbildung in einer anderen Stadt gemacht habe. (AB 1/26).

2) Beim Lachen werden im Körper viele in Bewegung gesetzt. $(\mathrm{AB} / 32)$

3) Dabei schüttet das Glückshormone aus. (AB/32)

In diesen Beispielen treten Kollokationen schon als eine Ganzheit auf, wobei ihre Kenntnis darin nicht abgefragt wird. Aus diesem Grund wurden diese Übungen nicht als Übungen zu Kollokationen eingestuft und jene Kollokationen (Beispiele 2 und 3), die früher in einem Lesetext vorkamen, der Gruppe der aus Lesetexten in Übungen transferierten Kollokationen nicht zugerechnet. Ähnlich war es auch mit der Kollokation einen Kredit aufnehmen (AB 7/22, siehe Beispielsatz 4).

\footnotetext{
${ }^{21}$ Schwer zu beurteilen ist der Gebrauch der Wortverbindung etwas in Bewegung bringen in diesem Text, denn die (normalerweise gelernte und in einsprachigen Wörterbüchern öfter vorkommende) Kollokation lautet in Bewegung setzen. $\mathrm{Ob}$ die in diesem Text auftretende Wortverbindung keine Kollokation oder überhaupt nicht korrekt gebraucht ist, ist den DaF-Lernenden nicht klar. Dies ist aus der Sicht der DaF-Lernenden insofern problematisch, als in einer sich auf diesen Text beziehenden Übung die Kollokation Muskeln in Bewegung setzen (AB 2/32) vorkommt.
} 

, hat er einen Kredit für den Hauskauf aufgenommen.

Diese wurde zwar in eine Übung zur Ergänzung der Sätze eingebettet. Da hier die Kollokation als eine Ganzheit angegeben wurde und die Lernenden sich auf den Inhalt des ersten Satzteils konzentrieren mussten, wurde diese Kollokation aber nicht in die Analyse mit einbezogen.

Problematisch zu bewerten war auch eine Übung, in der zwar viele Kollokationen angegeben werden (AB 3/31), aber die Aufgabe der DaF-Lernenden besteht dort darin, zu entscheiden, welche dieser Syntagmen sich auf die Tätigkeiten des Arztes und welche auf den Patienten beziehen. Hier werden folgende Kollokationen vermittelt: den Blutdruck messen, ein Medikament einnehmen, eine Diagnose stellen, den Oberkörper frei machen, ein Medikament verschreiben, ein Rezept ausstellen, einen Zahn ziehen. Diese Übung kann jedoch als ein Hinweis auf Kollokationen aufgefasst werden und kann zur Verbesserung der produktiven Beherrschung der Kollokationen dienen. Aus diesem Grunde wurde sie in der Analyse mit berücksichtigt.

Bei der Untersuchung der Lehrwerke wurde darauf geachtet, inwieweit darin die DaFLernenden zum kollokationsentdeckenden Lesen angeregt werden. Im Allgemeinen ist festzuhalten, dass diese Art des Umgangs mit Kollokationen nicht realisiert wird. Nur an einer Stelle finden wir ein Anzeichen der textbasierten Arbeit an Kollokationen. Es handelt sich dabei um eine Übung, in der die Kollokation Schmerzen vertreiben behandelt wurde. Diese wurde in eine Übung zur Zusammenfassung des Textes eingeflochten (AB 2/32). Darin muss der DaF-Lernende passende Wörter (verschiedene Wortarten, aber darunter auch den Kollokator) aus dem Lesetext exzerpieren und diese sinnvoll in den Lückentext einsetzen. Diese Übung kann als ein Schritt in Richtung zum kollokationsentdeckenden Lesen und zur Förderung des Kollokationsbewusstseins interpretiert werden. Zu bedauern ist, dass hier nur eine einzige Kollokation thematisiert wurde, obwohl in dem Ausgangslesetext, der zusammenzufassen war, mehr Kollokationen auftraten.

Darüber hinaus interessierte uns die Art und die Qualität der Übungen, in die zum einen Kollokationen aus den Texten transferiert wurden, in denen zum anderen neue Kollokationen behandelt wurden. Die Analyse hat ergeben, dass Kollokationen aus Lesetexten in Zuordnungsübungen auf der Wortebene sowie in grammatische Übungen eingebettet wurden. Die Anzahl der Übungen, in denen neue Kollokationen eingeführt wurden, ist nicht hoch. In solchen Übungen werden in der Regel einige Kollokationen behandelt. Hier überwiegt die Zuordnungsübung auf der Wortebene, in der einem Substantiv (Kollokationsbasis) ein Verb (Kollokator) zugeordnet werden muss. In diesem Lehrwerk sind jedoch auch andere vereinzelte Übungen anzutreffen, in denen manche Wörter ergänzt werden (Lückentexte, Ergänzungsübungen) oder vorgegebene Wörter bzw. Syntagmen in bestimmte Spalten eingeordnet werden müssen. Diese Übungen konzentrieren sich meistens auf die Form der Kollokationen. Inhaltsorientierte Übungen stellen hier eine Seltenheit dar.

Zusammenfassend ist festzustellen, dass in diesem Lehrwerk 138 Kollokationen gefunden wurden. Sie sind in den jeweiligen Kapiteln unterschiedlich verteilt. Davon treten 96 Kollokationen in Lesetexten auf, aber nur $9(9,4 \%)$ von ihnen wurden weiterhin in lexikalische Übungen integriert, weswegen sie von DaF-Lernenden geübt werden konnten. Dadurch wurde die Chance, dass die DaF-Lernenden diese internalisieren, anders als im Falle der Kollokatio- 
nen, die in Wortschatzübungen keine Berücksichtigung fanden, erhöht. Im Allgemeinen kann festgestellt werden, dass die Möglichkeit, anhand der Lesetexte neue Kollokationen einzuführen und sie den DaF-Lernenden bewusst zu machen, in diesem Lehrwerk nicht genutzt wurde, denn nur etwa 9,4\% dieser Kollokationen stellten den Übungsgegenstand dar. Dieses Manko kann nur zum Teil dadurch ausgeglichen werden, dass Kollokationen auch in verschiedenen Übungen, darunter insbesondere in Wortschatzübungen, eingeführt wurden. 43 neue Kollokationen wurden auf diese Art und Weise, d. h. ohne textuelle Einbettung eingeführt, wobei dies nur in drei Kapiteln (Kap. 2, 3 und 6) in größerer Menge erfolgte. Festzuhalten ist jedoch die Tatsache, dass fast $70 \%$ der Kollokationen, mit denen DaF-Lernende konfrontiert werden, in Lesetexten auftreten. Fast $30 \%$ neuer Kollokationen werden erst in lexikalischen Übungen eingeführt.

\section{$5.4 \quad$ Kollokationen in Sicher B1+}

Im Folgenden beginnen wir auch die Analyse dieses Lehrwerks mit einer kurzen Beschreibung seiner Charakteristik. Dieses ist inhaltlich weniger umfangreich als das oben analysierte Lehrwerk. Es besteht aus 8 Kapiteln mit zum Teil gleicher Thematik wie in Aspekte B1+. Die Anzahl der Lesetexte ist im Vergleich zum Lehrwerk Aspekte B1+ geringer und diese sind viel kürzer. Ihre Menge schwankt in den einzelnen Kapiteln zwischen drei und sechs, wobei in jedem Kapitel durchschnittlich vier Texte zu finden sind, von denen nur einer oder zwei relativ lang sind. Die weiteren Texte sind kurz und viele von ihnen (insbesondere im Arbeitsbuch) sind Lückentexte. Sie dienen also dem formorientierten Lesen, wobei dort nicht Kollokationen, sondern andere Vokabeln bzw. grammatische Formen ergänzt werden sollten. Texte in diesem Lehrwerk sind nicht reich an Kollokationen, was der Tabelle 5 zu entnehmen ist.

\begin{tabular}{|l|c|c|c|}
\hline Kapitel & $\begin{array}{l}\text { Anzahl der in Lesetexten } \\
\text { vorkommenden Kolloka- } \\
\text { tionen }\end{array}$ & $\begin{array}{l}\text { Anzahl dieser Kollokationen (aus } \\
\text { Lesetexten), die in lexikalischen } \\
\text { Übungen berücksichtigt wurden }\end{array}$ & $\begin{array}{l}\text { Anzahl der erst in Wort- } \\
\text { schatzübungen eingeführ- } \\
\text { ten Kollokationen }\end{array}$ \\
\hline Kap. 1 & 3 & 1 & 2 \\
\hline Kap. 2 & 3 & 0 & 3 \\
\hline Kap. 3 & 7 & 0 & 0 \\
\hline Kap. 4 & 3 & 0 & $8(2)^{22}$ \\
\hline Kap. 5 & 5 & 0 & 0 \\
\hline Kap.6 & 6 & 0 & 11 \\
\hline Kap.7 & 4 & 0 & $\mathbf{3 6}$ \\
\hline Kap. 8 & 1 & $\mathbf{2}$ & 11 \\
\hline Insgesamt & $\mathbf{3 2}$ & 0 & 1 \\
\hline
\end{tabular}

Tabelle 5: Kollokationen in Lesetexten und in lexikalischen Übungen in Sicher B+

Aus der quantitativen Analyse der Lesetexte und Wortschatzübungen unter dem Aspekt des Auftretens bzw. der Berücksichtigung von Kollokationen ergibt sich, dass die Anzahl der in Lesetexten vorkommenden Kollokationen sehr gering ist und nur 32 Kollokationen beträgt (vgl. Tabelle 5). In den Lesetexten dieses Lehrwerks kommen durchschnittlich vier Kolloka-

\footnotetext{
${ }^{22}$ In lexikalischen Übungen wurden zwar acht neue Kollokationen eingeführt, aber zwei davon wurden zweimal zum Übungsgegenstand gemacht.
} 
tionen in jedem Kapitel vor, wobei in fünf Kapiteln ihre Anzahl nicht höher als drei ist. Somit bilden diese Texte keinen guten Ausgangspunkt für den Transfer der Kollokationen aus Lesetexten in Übungen. Obwohl nur zwei von den 32 textuell eingebetteten Kollokationen Widerspiegelung in Wortschatzübungen fanden, ist der Prozentsatz der aus diesen Lesetexten in Wortschatzübungen transferierten und zum Übungsgegenstand gemachten Kollokationen nur ein bisschen kleiner als in Aspekte B1 + und beträgt 6,25 \%. Die geringe Anzahl der aus Lesetexten transferierten Kollokationen kann daraus resultieren, dass im Lehrbuch zwar textbezogene Übungen auftreten, sich diese aber vorwiegend auf den Inhalt (Suche nach Informationen, R/F-Aufgaben) beziehen bzw. zum Kommunizieren anregen. Es gibt vereinzelte formfokussierende textbezogene Übungen, aber diese konzentrieren sich auf die Grammatik (Rektion des Verbs, Vorsilben bzw. präfigierte Verben, Tempusgebrauch). Die in Lesetexten vorkommenden Kollokationen werden in der Regel in lexikalischen Übungen nicht aufgegriffen.

Kollokationen, die in Lesetexten dieses Lehrwerks vorkommen, sind Kollokationen des alltäglichen Gebrauchs wie z. B. Angst haben, eine Wanderung machen, ins Gespräch kommen, Fragen stellen, PIN eingeben, Probleme lösen, Spaß machen, Erfahrungen sammeln, Geld ausgeben usw. Kollokationsreiche Texte sind in diesem Lehrwerk nicht zu finden. Die meisten Texte mit Kollokationen enthalten eine oder zwei solche konventionellen Wortverbindungen. Nur in einem Text (,Mobilität in der Großstadt“, AB S. 38) sind sechs Kollokationen zu finden (den Bus benutzen, Wege mit dem Bus zurücklegen, Fragen stellen, die Stadt erkunden, die PIN eingeben, das Schloss entriegeln). Dieser stellt eine Ausnahme dar. Nur zwei von den oben genannten Kollokationen wurden aus den Lesetexten exzerpiert und in Wortschatzübungen eingebettet. Dabei handelt es sich um die Kollokationen eine wichtige Rolle spielen und Verantwortung übernehmen. Eine Erörterung verdient die Tatsache, dass diese Kollokationen nicht nur in einer Wortschatzübung ihren Platz gefunden haben. Die erste Kollokation wurde in zwei Übungen berücksichtigt, und zwar in einer Zuordnungsübung, in der Substantiven (darunter auch Kollokationsbasen) passende Verben (darunter auch der Kollokator) zugeordnet werden sollten (AB 21/16) (vgl. Graphik 4), und in einer Übung, in der zu einem Substantiv nicht passende Verben (Kollokatoren) erkannt werden müssen (AB 6/120). Diese Übungen (die erste auf der Wort- und die zweite auf der Satzebene) sind formorientiert, d. h. sie lenken die Aufmerksamkeit der DaF-Lernenden auf die Form der Kollokationen, insbesondere auf den Kollokator.

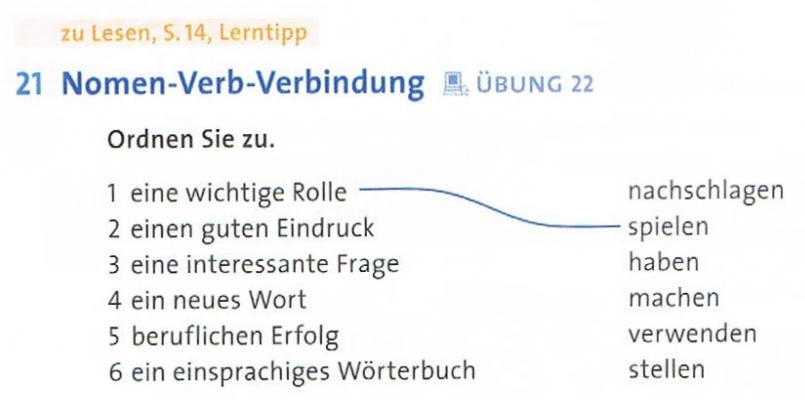

Graphik 4: Übungen zur Einführung und Bewusstmachung von syntagmatischen Wortverbindungen in Sicher B1+ (AB, S. 16)

Überraschend ist die Tatsache, dass die zweite oben erwähnte Kollokation Verantwortung übernehmen sogar in drei unterschiedliche Übungen integriert wurde. Im Unterschied zu der ersten aus dem Lesetext in Wortschatzübungen transferierten Kollokation ist diese in andere 
Übungsformen eingebettet. Zuerst tritt sie in einer Übung zur Textzusammenfassung auf, in der in einem kurzen kohärenten Text Verben (darunter auch der Kollokator) ergänzt werden müssen (diese Verben stehen den DaF-Lernenden zur Wahl [AB 23/78]) (vgl. Graphik 5). Im Anschluss daran befindet sich eine Übung, in der die Aufmerksamkeit der DaF-Lernenden auf die Bedeutung dieser Kollokation und anderer Syntagmen gelenkt wird (AB 24/78). Zum dritten Mal wird diese Kollokation in dem Lektionstest zum Kapitel 5 berücksichtigt (AB 1/84), in dem zu bestimmten Substantiven drei unterschiedliche Verben zur Wahl stehen, wobei das korrekte Verb erkannt werden soll.



Graphik 5: Berücksichtigung der Kollokation Verantwortung übernehmen in Wortschatzübungen (Sicher B1+, AB S. 78)

Interessant ist in diesem Lehrwerk die Tatsache, dass hier viele Kollokationen, die in Texten nicht vorkamen, in Übungen zum Wortschatz eingeführt und gleichzeitig geübt werden. Auf diese Weise werden DaF-Lernende darin auf Kollokationen und andere syntagmatische Wortverbindungen aufmerksam gemacht. Dies erfolgt in fast allen Kapiteln.

Bezüglich der Art der Übungen, in denen Kollokationen den Übungsgegenstand darstellen, ist festzustellen, dass Kollokationen oft in Übungen zur Wortsyntagmatik integriert werden. Die meisten werden in Übungen zu Kapitel 5 eingeführt und eingeübt. Dabei handelt es sich um folgende Kollokationen: einen Arbeitsvertrag unterschreiben, eine Ausbildung machen, ein Ziel erreichen, Geld verdienen, Geld ausgeben, eine Schule (ein Gymnasium) besuchen, eine (Berufs)ausbildung machen. In lexikalischen Übungen zum Kapitel 7 kommen im Arbeitsbuch folgende Kollokationen vor: einen Preis vergeben, eine Karte ziehen, Geld sparen, Geld ausgeben, ein Konto eröffnen, ein Konto überziehen, ein Konto sperren, Geld abheben, Geld ausgeben, Geschirr abwaschen. In Kapitel 8 werden DaF-Lernende mit den Kollokationen eine Prüfung bestehen, ein Formular ausfüllen, einen (Volkshochschul)Kurs besuchen, eine 
CD brennen, Filme drehen, einen Computer hochfahren, ein Computerprogramm herunterladen, ein Computerprogramm aufrufen, eine E-Mail weiterleiten konfrontiert.

Uns interessiert auch die Qualität und Vielfalt der Übungsformen, in denen neue Kollokationen eingeführt werden und die dort den Übungsgegenstand darstellen. Die häufigste Form ist eine Zuordnungsübung auf der Wortebene, in der einem Substantiv (z. B. einer Kollokationsbasis) ein Kollokator zugeordnet werden muss (vgl. Graphik 4). Diese Übungsform ist auch, obwohl relativ selten, im Lehrbuch zu finden.

Eine weitere Übungsform ist auch eine formfokussierende Übung, in der zu einem Substantiv passende bzw. unpassende Verben (bzw. Kollokatoren) erkannt werden müssen (vgl. Graphik $6)$.

\section{Ausbildung und Beruf 欥 ÜBUNG 1}
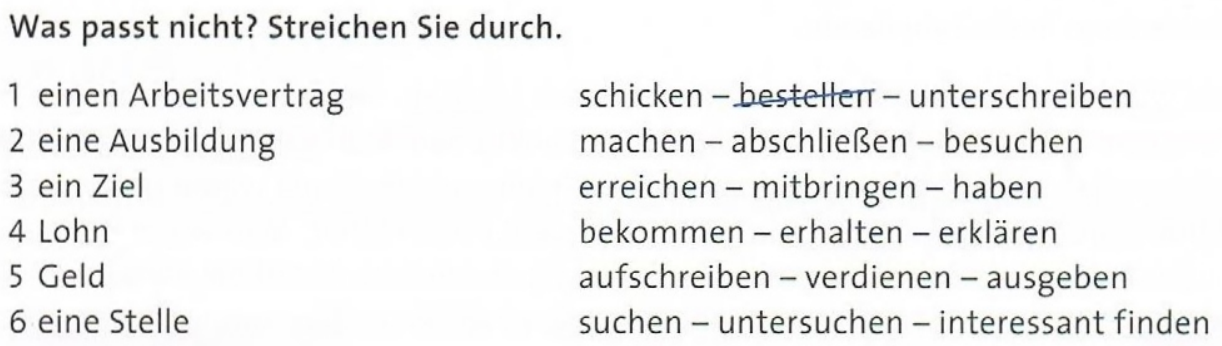

Graphik 6: Übungen zur Einführung und Bewusstmachung von syntagmatischen Wortverbindungen in Sicher B1 + (AB 2/69)

In diesen beiden Übungstypen müssen korrekte bzw. unkorrekte syntagmatische Partner erkannt werden, wobei diese schon zur Wahl stehen. Aber ausnahmsweise müssen den Verben auch passende Substantive zugeordnet werden (AB 2/117). Diese Übungen prüfen die rezeptive Kollokationskompetenz, weil die diese Übung machenden DaF-Lernenden den Kollokator nicht selbstständig angeben müssen. Halbproduktive Übungen, in denen die Kenntnis der Kollokationen geprüft wird, sind Lückentexte, in denen Verben oder Substantive ergänzt werden müssen.

Erörterungswert ist noch eine Art von Übungen, in die Kollokationen integriert wurden. Darin werden sog. Kollokationsreihen erstellt, d. h. die Lernenden müssen bestimmen, welche Verben (darunter Kollokatoren) zu einem Substantiv (auch als Kollokationsbasis) passen (vgl. Graphik 7 und 8). Dank diesem Übungstyp können sich Fremdsprachenlernende der horizontalen und vertikalen Ebene der Kollokationen (cf. Irsula Peña 1996) bewusst werden. Diese Übungsform kann zur Förderung des Kollokationsbewusstseins eingesetzt werden. 
b Was passt nicht? Streichen Sie das Wort durch.

\begin{tabular}{|l|l|l|l|}
\hline einen Computer ... & eine Datei ... & eine E-Mail ... & ein Computerprogramm ... \\
\hline anschließen & öffnen & tippen & aufrufen \\
hochfahren & ausdrucken & einlegen & herunterladen \\
verbinden & löschen & versenden & aufschließen \\
einlegert & anschließen & weiterleiten & schließen \\
\hline
\end{tabular}

\begin{tabular}{|l|l|l|l|}
\hline eine CD-ROM ... & Lautsprecher ... & auf einem USB-Stick ... & mit einer Web-Cam etwas ... \\
\hline vergrößern & leise drehen & speichern & filmen \\
einlegen & anschließen & kopieren & drucken \\
brennen & lauter machen & surfen & aufnehmen \\
anklicken & abschließen & löschen & sehen \\
\hline
\end{tabular}

c Wie ist Ihr Arbeitsplatz ausgestattet?

Was brauchen Sie unbedingt?

Für mich ist mein USB-Stick ganz wichtig. Den brauche ich, wenn..

Graphik 7: Eine Übung zur Bewusstmachung der vertikalen Ebene der Kollokationen (Übung zur Erstellung der Kollokationsreihen) in Sicher B1+ (KB 1b/96)

17 Geld 或 ÜBUNG 22

WORTSCHATZ

a Was kann man alles mit einem Konto, Geld und Münzen machen? Ordnen Sie zu.

sparen - überweisen - übrig haben - bezahlen $\cdot$ ausgeben - aufder Bank haben verlieren • leihen · dabeihaben • kündigen - eröffnen • abheben • wechseln • umtauschen - sammeln • auf dem Konto haben • überziehen • sperren

1 ein Konto: anf der Bank haben, eröffuen, ...

2 Geld:

3 Münzen:

b Schreiben Sie Sätze.

1 Ich habe ein Konto anf der Bank. Gestern habe ich ein Konto eröffnet.

Graphik 8: Übung zur Erstellung der Kollokationsreihen in Sicher B1+ (AB 17/108)

Im Allgemeinen kann festgestellt werden, dass die in diesem Lehrwerk auftretenden Texte wenige Kollokationen enthalten. Diese werden oft nicht in Übungen eingebettet und den Lernenden auch nicht bewusst gemacht. Dieser Nachteil wird zum Teil durch vorwiegend lexikalische Übungen ausgeglichen, in denen Kollokationen erst eingeführt und mitgeübt werden. Überraschend kann hier die Tatsache sein, dass in Sicher B1+ ein bisschen mehr Kollokationen erst in Wortschatzübungen (36) eingeführt werden als in Lesetexten. Dort konnten 32 Kollokationen ausfindig gemacht werden.

Beim Vergleich der beiden Lehrwerke fällt auf, dass darin die textbasierte Kollokationsdidaktik im Sinne ,vom Text zur Übung“ nicht realisiert wird, weswegen textbasiertes Kollokationslernen nicht zustande kommt. Zwar enthalten Texte im Lehrwerk Aspekte B1+ wesentlich mehr Kollokationen als in Sicher B1+ (dies kann an der größeren Anzahl der Lesetexte und ihrer Länge liegen), aber diese Kollokationen werden weiterhin in lexikalischen bzw. kommunikativen Übungen nur selten aufgegriffen und zum Übungsgegenstand gemacht. 


\subsection{Zusammenfassung der Ergebnisse der Analyse von Lehrwerken}

Die oben dargestellte Analyse der beiden DaF-Lehrwerke für die Niveaustufe B1+ erlaubt die Beantwortung der oben (siehe 5.1) formulierten Forschungsfragen, was im folgenden $\mathrm{Ab}$ schnitt erfolgt. Im Allgemeinen kann festgestellt werden, dass die beiden Lehrwerke die textbasierte Arbeit nicht realisieren. Aus der Untersuchung geht hervor, dass die in Lesetexten vorkommenden Kollokationen in der Regel in Wortschatzübungen nicht transferiert werden, d. h. sie werden in diesen Übungen nicht zum Übungsgegenstand gemacht. Nur ca. $6 \%-9 \%$ der Kollokationen, mit denen DaF-Lernende im Leseprozess der Texte konfrontiert werden, werden weiterhin in Wortschatzübungen explizit behandelt. Wenn gegen 91\%-94\% der in Lesetexten auftretenden Kollokationen keine Widerspiegelung in Wortschatzübungen finden, so müssen diese $6 \%$ bzw. $9 \%$ der berücksichtigten Kollokationen als eine Ausnahme von der Regel betrachtet werden. Somit muss die erste Forschungsfrage (Werden die in Lesetexten auftretenden Kollokationen in Wortschatzübungen aufgegriffen und zum Übungsgegenstand gemacht?) verneint werden.

Die zweite und dritte Forschungsfrage bezüglich der Förderung des kollokationsentdeckenden Lesens und des Kollokationsbewusstseins, die zum Teil aus der ersten resultieren, müssen auch verneint werden. Im Allgemeinen werden die Lernenden in beiden Lehrwerken nicht dazu angehalten, nach Kollokationen in Lesetexten zu suchen. Dies bewirkt, dass in diesen Lehrwerken das Kollokationsbewusstsein nicht explizit gefördert wird, obwohl dieses auch von der Lehrkraft, ihrem Umgang mit den Texten und der von ihr betriebenen Wortschatzarbeit doch gefördert werden kann. Uns interessierte jedoch das Lehrwerk selbst. Somit müssen die zweite Frage (Werden die Lernenden durch die lexikalischen Übungen zum formorientierten Lesen der Texte ermuntert bzw. angeregt?) und die dritte (Können mittels der [lexikalischen] Übungen kollokationsentdeckendes Lesen und Kollokationsbewusstsein gefördert werden?) ebenfalls verneint werden. Erörterungswert ist jedoch, dass in beiden analysierten Lehrwerken jeweils eine Übung, in der textbasierte Arbeit an Kollokationen betrieben wird, $\mathrm{zu}$ finden ist. Es handelt sich dabei um eine lexikalische, sowohl form- als auch bedeutungsfokussierende Übung, in der ein Lesetext zusammengefasst werden muss, wobei eine nicht komplette Zusammenfassung mit Lücken durch bestimmte aus dem Text exzerpierte lexikalische Einheiten zu ergänzen ist.

Die vierte Forschungsfrage bezog sich auf den Anteil der aus Lesetexten exzerpierten und zum Übungsgegenstand gemachten Kollokationen. Die Anzahl der Kollokationen die „vom Text zur Übung“ transferiert wurden, variiert je nach dem Lehrwerk. In Aspekte B1+ wurden in Lesetexten 95 Kollokationen ausfindig gemacht, von denen nur 9 (d. h. 9,4\%) in Wortschatzübungen explizit behandelt wurden. In Lesetexten des Lehrwerks Sicher B1+ wurden nur 32 Kollokationen ermittelt. Davon wurden ausschließlich 2 (also auch etwa $6 \%$ ) zum Übungsgegenstand gemacht. Darin sind sich die beiden Lehrwerke ähnlich.

Einer genaueren Beschreibung bedarf die Beantwortung der fünften Forschungsfrage nach der Art und Qualität der Übungen, in denen Kollokationen geübt werden. Dies kann auch aus zwei Perspektiven betrachtet werden. Zum einen wird hier gezeigt, in welche Übungsformen die $6 \%-9 \%$ der aus Lesetexten exzerpierten und zum Übungsgegenstand gemachten Kollokationen eingebettet wurden. Zum anderen kann hier gezeigt werden, in welcher Art von Übungen bzw. Aufgaben ,neue“ Kollokationen behandelt werden. Im Lehrwerk Aspekte B1+ 
wurden 9 Kollokationen aus Lesetexten zum Übungsgegenstand gemacht, wobei zwei von ihnen in einzelne Wortschatzübungen integriert, zwei in jeweils zwei lexikalische Übungen und zwei in kommunikative Übungen eingebettet wurden. Die Kollokation sich eine Auszeit gönnen wurde in eine inhaltsorientierte Übung eingeflochten, in der dem jeweiligen Phrasem bzw. der Kollokation die passende Bedeutung zugeordnet werden muss (KB 3b/17). Die oben erwähnte Kollokation Schmerzen vertreiben wurde in einer textbasierten Übung zum kollokationsentdeckenden Lesen behandelt (vgl. AB 2/32). Diese Kollokation wurde noch in eine weitere Übung eingeflochten, und zwar in eine grammatische, in der Präfixe der präfigierten Verben (darunter Kollokatoren) ergänzt werden müssen. In dieser Übung wird die Aufmerksamkeit der Lernenden auf die Form des Kollokators gelenkt.

Die beiden aus Lesetexten exzerpierten Kollokationen wurden im Lehrwerk Sicher B1+ in unterschiedliche Übungsformen eingebettet. Diese Vielfalt resultiert daraus, dass die beiden Kollokationen in zwei oder drei Übungen wieder aufgegriffen wurden. Die Kollokation Verantwortung übernehmen wurde sowohl in einer formfokussierenden als auch in einer auf den Inhalt ausgerichteten Übungsform zum Übungsgegenstand gemacht. In der Übung (AB 23/78) muss ein Lückentext mit bestimmten Wörtern (darunter u. a. mit dieser einzigen Kollokation) ergänzt werden. Die darauf folgende Übung (AB 24/78) ist darauf ausgerichtet, einer lexikalischen Einheit bzw. Phrase ihre Bedeutung zuzuordnen. Form- und inhaltsfokussierend ist die dritte Übung im Lektionstest (AB 1/84), wo in einem kohärenten Text eines der drei zur Verfügung stehenden Verben gewählt werden muss. Der DaF-Lernende, der diese Übung macht, muss sich auf den Inhalt konzentrieren, um anhand des Textverstehens das korrekte Verb bzw. den korrekten Kollokator zu wählen. Diese Behandlung der Kollokationen kann als musterhaft bezeichnet werden. Die zweite aus dem Lesetext in eine Übung transferierte Kollokation (eine Rolle spielen) wurde sowohl in eine formfokussierende Zuordnungsübung eingebettet, in der dem Substantiv (Basis) das passende Verb (der Kollokator) zugeordnet werden muss, als auch in einer Übung behandelt, in der in den angegebenen Sätzen nicht passende (von drei angegebenen) Verben durchgestrichen werden müssen (AB 6/120). Diese beiden Übungen fokussieren zwar die Aufmerksamkeit der Lernenden auf die Form der Kollokationen, jedoch wird hier nur die rezeptive Kollokationskompetenz gefördert.

In Bezug auf die siebte Forschungsfrage (Fokussieren diese Übungen, in denen Kollokationen auftreten, ihre Form bzw. ihre Bedeutung?) ist festzuhalten, dass in Übungen, in denen Kollokationen mitgeübt werden, die Aufmerksamkeit der Lernenden viel stärker auf die Form und nur ausnahmsweise auf die Bedeutung der Kollokationen gelenkt wird. Hier ist noch anzumerken, dass mit diesen Übungen keine korrekte Kollokationsdidaktik betrieben wird. Die Übungsphase geht nur selten in eine Anwendungsphase über.

\section{$6 \quad$ Fazit und didaktische Implikationen}

Die in der vorliegenden Studie dargestellten Ergebnisse der Lehrwerkanalyse unter dem Aspekt der Behandlung von Kollokationen zeigen, dass in beiden untersuchten Lehrwerken die textbasierte Arbeit an Kollokationen nicht betrieben wird. Aus den Resultaten dieser Untersuchung lassen sich bestimmte Konsequenzen bzw. didaktische Implikationen ableiten. Diese beziehen sich sowohl auf DaF-Lehrwerke als auch auf DaF-Lehrkräfte. 
DaF-Lehrwerke sollten zwar auf allen Niveaustufen die Kollokabilität der deutschen Sprache berücksichtigen und diese explizit zum Übungsgegenstand machen. Aber je höher das sprachliche Niveau der DaF-Lernenden, desto öfter sollte man zur textbasierten Arbeit an Kollokationen greifen. Bezüglich des kollokationalen Lernens ist von den DaF-Lehrwerken zu erwarten, dass diese mehr textbasierte Kollokationsübungen enthalten, dank denen DaF-Lernende zum kollokationsentdeckenden Lesen angeregt werden könnten. Diese sollten die Form der Kollokationen bzw. deren Bedeutung fokussieren. Wünschenswert wäre auch eine gut durchdachte Reihenfolge der Übungen, die von der Wortebene ausgehen, über die Satzebene gehen und bis zur Textebene kommen sollten. Die Arbeit an Kollokationen sollte also von Texten ausgehen und wieder zu Texten führen. Dies bedeutet, dass formfokussierende Übungen nur den Ausgangspunkt darstellen sollten. Wichtig ist der halbproduktive und der produktive Gebrauch von Kollokationen, dem weitere Übungen gewidmet werden sollten. Wenn DaFLernende Kollokationen dauerhaft speichern sollten, ist die spiralförmige Behandlung der Kollokationen notwendig. Diese äußert sich darin, dass eine einmal eingeführte Kollokation nach einer bestimmten Zeit wieder aufgegriffen werden (Festigungs- bzw- Wiederholungsübungen) und das nächste Mal den Übungsgegenstand darstellen muss. Nicht zu vergessen sind auch kommunikative Übungen zum Gebrauch von Kollokationen, die ihren Platz in den Lehrwerken finden sollten.

Zwar steuert das Lehrwerk sehr stark das Unterrichtsgeschehen, aber auch DaF-Lehrkräfte haben ihre nicht zu unterschätzende Rolle bei der Förderung der Kollokationskompetenz. Diese sollten nicht nur das Lehrwerk „,realisieren“, d. h. Seite nach Seite ,abhaken“, sondern auch den Unterricht so führen, dass ihre Schüler ihre Wortschatzkompetenz bzw. kommunikative Kompetenz vollständig entfalten können. Die Aufgabe der Lehrkraft besteht in erster Linie darin, den DaF-Lernenden die Kollokabilität der (deutschen) Sprache bewusst zu machen. Weiterhin sollten Kollokationen in der Wortschatzarbeit sowie auch in der Arbeit an Texten berücksichtigt werden. Die DaF-Lehrkräfte sollten bei der Wortschatzeinführung und -semantisierung auf Kollokationen hinweisen. Diese sollten aber auch in der Übungs- und insbesondere in der Anwendungsphase ihren Platz finden.

Betonenswert ist ebenso die Tatsache, dass DaF-Lehrkräfte als Spezialisten für die deutsche Sprache fungieren (sollten). Auf der einen Seite beurteilen sie, ob die im Lehrwerk auftretenden Kollokationsübungen von DaF-Lernenden korrekt gemacht wurden, denn nicht in jedem Lehrwerk gibt es einen Lösungsschlüssel, mit dem diese die Ergebnisse ihres lexikalischen Übens selbstständig prüfen könnten. Auf der anderen Seite kann die DaF-Lehrkraft auch erklären, was korrekt und was nicht korrekt ist bzw. was unter welchen Voraussetzungen akzeptabel sein könnte.

Die Lehrkraft, die im Gegensatz zu Lehrwerkautoren ihre DaF-Lernenden kennt, sollte die mit Lehrwerken betriebene Arbeit durch andere Arbeitsformen und zusätzliche Materialien ergänzen. Da, wie aus den oben dargestellten Untersuchungsergebnissen hervorgeht, Lehrwerke viele Nachteile hinsichtlich der Arbeit an Kollokationen und der Förderung der Kollokationskompetenz aufweisen, sollte die Lehrkraft bei ihrer Textarbeit auch Kollokationen berücksichtigen. Dies bedeutet, dass sie DaF-Lernende zum kollokationsentdeckenden Lesen anregen sollte. Dabei besteht ihre Aufgabe darin, zu Texten selbstständig Arbeitsblätter zu erstellen, dank denen die Aufmerksamkeit der DaF-Lernenden auf die Form des Kollokators 
gelenkt wird. Nicht zu vergessen ist auch die Konzipierung kommunikativer Aufgaben zur Anwendung von neu kennengelernten Kollokationen. Zu der selbstständigen Erstellung von Zusatzmaterialien muss die DaF-Lehrkraft selbst über Kollokationswissen, Kollokationsbewusstsein verfügen und gut entwickelte Kollokationskompetenz haben.

\section{Literatur}

\section{Primärliteratur (Untersuchte Lehrwerke)}

Koithan, Ute et al. (2007a): Aspekte. Mittelstufe Deutsch. Lehrbuch 1. Berlin/München: Langenscheidt.

Koithan, Ute et al. (2007b): Aspekte. Mittelstufe Deutsch. Arbeitsbuch 1. Berlin/München: Langenscheidt.

Perlmann-Balme, Michaela/Schwalb, Susanne (2012): Sicher! B1+ Deutsch als Fremdsprache. Kursbuch. Ismaning: Hueber.

Orth-Chambah, Jutta/Perlmann-Balme, Michaela/Schwalb, Susanne (2012): Sicher! B1+ Deutsch als Fremdsprache. Arbeitsbuch. Ismaning: Hueber.

\section{Sekundärliteratur}

Bahns, Jens (1993): „Kollokation kontra Kontext. Wider ein zu weites Verständnis des Kollokationsbegriffs“". Praxis des Neusprachlichen Unterrichts 40/1: 30-37.

Bahns, Jens (1997): Kollokationen und Wortschatzarbeit im Englischunterricht. Tübingen: Narr.

Bahns, Jens/Sibilis, Ulrich (1992): „Kollokationslernen durch Lektüre“. Neusprachliche Mitteilungen aus Wissenschaft und Praxis 45/3: 158-163.

Burger, Harald (2007): Phraseologie. Eine Einführung am Beispiel des Deutschen. 3. Auflage. Berlin: E. Schmidt.

Caro Cedillo, Anna (2004): Fachsprachliche Kollokationen. Ein übersetzungsorientiertes Datenbankmodell. Deutsch-Spanisch. Tübingen: Narr.

Coseriu, Eugenio (1967): „Lexikalische Solidaritäten“. In: Geckeler, Horst (ed.) (1978): Strukturelle Bedeutungslehre. Darmstadt, Wissenschaftliche Buchgesellschaft: 239-253.

Ettinger, Stefan (2007): „Phraseme im Fremdsprachenunterricht“. In: Burger, Harald et al. (eds.): Phraseologie. Phraseolgy. Ein internationales Handbuch der zeitgenösischen Forschung. Halbband 2. Berlin/New York, de Gruyter: 893-908. (= HSK 28.2).

Ettinger, Stefan (2011): „Einige kritische Fragen zum gegenwärtigen Forschungsstand der Phraseodidaktik“. In: Schäfer, Patrick/Schowalter, Christine (eds.): In mediam Linguam: Mediensprache - Redewendungen - Sprachvermittlung. Landau, Verlag Empirische Pädagogik: 231-250.

Ettinger, Stefan (2013): „Aktiver Phrasemgebrauch und/oder passive Phrasemkenntnisse im Fremdsprachenunterricht. Einige phraseodidaktische Überlegungen“. In: González Rey, Isabel (ed.): Phraseodidactic Studies on German as a Foreign Language. Phraseodidaktische Studien zu Deutsch als Fremdsprache. Hamburg, Dr. Kovač: 11-30. (= Lingua. Fremdsprachenunterricht in Forschung und Praxis 22).

Forkl, Yves (2010): Zur digitalen Zukunft der Kollokationslexikographie. Perspektiven der Präsentation von Wissen über usuelle französische und deutsche Wortverbindungen in ge- 
druckten und elektronischen Wörterbüchern. Erlangen-Nürnberg: Friedrich-AlexanderUniversität Erlangen-Nürnberg. https://opus4.kobv.de/opus4-fau/frontdoor/index/index/ docId/1497 [31.03.2017].

Gabryś-Biskup, Danuta (1990): "Some remarks on combinability: Lexical collocation". In: Arabski, Jan (ed.): Foreign language acquisition papers. Katowice, Uniwersytet Śląski: 31-44.

Gładysz, Marek (2003): Lexikalische Kollokationen in deutsch-polnischer Konfrontation. Frankfurt a. M. etc.: Lang.

Häcki Buhofer, Annelies (2011): „Lexikographie der Kollokationen zwischen Anforderungen der Theorie und der Praxis“. In: Engelberg, Stefan/Holler, Anke/Proost, Kristel (eds.): Sprachliches Wissen zwischen Lexikon und Grammatik. Berlin/Boston, de Gruyter: 505531.

Hartenstein, Klaus (1991): „Die Vermittlung von Lexemkollokationen im Russischunterricht im Lichte der neueren phraseologischen Forschung“. In: Hartenstein, Klaus/Jachnow, Helmut (eds.): Slavistische Linguistik 1990 - Referate des XVI. Konstanzer Slavistischen Arbeitstreffens Bochum/Löllinghausen 18.-21.9.1990. München, Sagner: 98-120. (= Slavistische Beiträge 274).

Hausmann, Franz Josef (1976): „Linguistik des Wortschatzlernens im Französischstudium“. Grazer Linguistische Studien 4: 49-60.

Hausmann, Franz Josef (1984): „Wortschatzlernen ist Kollokationslernen. Zum Lehren und Lernen französischer Wortverbindungen“. Praxis des neusprachlichen Unterrichts 3/4: 395-406.

Hausmann, Franz Josef (1985): „Kollokationen in deutschen Wörterbüchern. Ein Beitrag zur Theorie des lexikographischen Beispiels“. In: Bergenholtz, Henning/Mugdan, Joachim (eds.): Lexikographie und Grammatik. Akten des Essener Kolloquiums zur Grammatik im Wörterbuch 28-30.06.1984. Tübingen, Niemeyer: 118-129. (= Lexicographica. Series Maior 3).

Hausmann, Franz Josef (2003): „Kollokationen in der Fachsprache: Schwerpunkt Französisch“. In: Jung, Udo O. H./Kolesnikova, Angelina (eds.): Fachsprachen und Hochschule. Frankfurt a. M. etc., Lang: 83-92. (= Bayreuther Beiträge zur Glottodidaktik 9).

Hausmann, Franz Josef (2004): „Was sind eigentlich Kollokationen?“. In: Steyer, Kathrin (ed.): Wortverbindungen - mehr oder weniger fest. Berlin, de Gruyter: 309-334.

Hausmann, Franz Josef (2007): „Die Kollokationen im Rahmen der Phraseologie - systematische und historische Darstellung". Zeitschrift für Anglistik und Amerikanistik 55/3: 217234.

Hollós, Zita (2010): „Auf dem schmalen Grad zwischen Kollokationen und festen Wortverbindungen“. In: Ďurčo, Peter (ed.): Feste Wortverbindungen. Kolloquium zur Lexikographie und Wörterbuchforschung. Berlin/New York, de Gruyter: 85-96.

Holzinger, Herbert Josef (1993): „Phraseologismen im Fremdsprachenunterricht“. Revista de Filología Moderna 4: 7-22.

Irsula Peña, Jesús (1994): Substantiv-Verb-Kollokationen. Kontrastive Untersuchungen Deutsch-Spanisch. Frankfurt a. M. etc.: Lang.

Konecny, Christine (2010a): Kollokationen. Versuch einer semantisch-begrifflichen Annäherung und Klassifizierung anhand italienischer Beispiele. München: Martin Meidenbauer. 
Konecny, Christine (2010b): „Lexikalische Kollokationen und der Beitrag der Internet Suchmaschine Google zu ihrer Erschließung und Beschreibung“. In: Ptashnyk, Stefaniya/Hallsteinsdóttir, Erla/Bubenhofer, Noah (eds.): Computergestützte und korpusbasierte Methoden in der Phraseologie, Phraseografie und der Lexikografie. Baltmannsweiler, Schneider Verlag Hohengehren: 77-93.

Kratochvílová, Iva (2004): „Usuelle Wortverbindungen: Fokussierung des aktuellen Themas ,Kollokabilität lexikalischer Einheiten' auf den Bereich Deutsch-Tschechischen Sprachkontrasts“. Brünner Beiträge zur Germanistik und Nordistik 18: 97-107.

Krenn, Brigitte (2004): Manual zur Identifikation von Funktionsverbgefügen und figurativen Ausdrücken in PP-Verb-Listen. www.collocations.de/guidelines/Krenn2000-Guide lines.pdf [10.03.2018].

Kühn, Peter (1992): „Phraseodidaktik. Entwicklungen, Probleme und Überlegungen für den Muttersprachenunterricht und den Unterricht Deutsch als Fremdsprache“. Fremdsprachen Lehren und Lernen 21: 167-189.

Laskowski, Marek (2004): „Toponymie im Bereich der phraseologichen Subsysteme des Deutschen und des Polnischen. Ein Beitrag zu ihrer konfrontativen Untersuchung und didaktischer Potenz". Info DaF 6: 596-616.

Laskowski, Marek (2010): „Phraseodidaktische Einsatzmöglichkeiten von Fernsehwerbung im DaF-Unterricht“. In: Korhonen, Jarmo et al. (eds.): Europhras 2008. Beiträge zur internationalen Phraseologiekonferenz vom 13.-16.08.2008 in Helsinki. Helsinki, Universität Helsinki: 399-409.

Leonhardi, Adolf (1964): „Die natürliche Spracheinheit“. Praxis des neusprachlichen Unterrichts 11: 17-22.

Ludewig, Petra (2005): Korpusbasiertes Kollokationslernen. Computer-Assisted Language Learning als prototypisches Anwendungsszenario der Computerlinguistik. Frankfurt a. M. etc.: Lang.

Lüger, Heinz-Helmut (1997): „Anregungen zur Phraseodidaktik“. Beiträge zur Fremdsprachenvermittlung 32: 69-120.

Lüger, Heinz-Helmut (2004): „Kollokationen - zwischen Arbitrarität und Kompositionalität“. In: Pohl, Inge/Konerding, Klaus-Peter (eds.): Stabilität und Flexibilität in der Semantik. Strukturelle, kognitive und historische Perspektiven. Frankfurt a. M. etc., Lang: 45-66.

Marton, Waldemar (1977): "Foreign vocabulary learning as a problem No. 1 of language teaching at the advanced level". Interlanguage Studies Bulletin 2: 33-57.

Nesselhauf, Nadja (2003): "The Use of Collocations by Advanced Learners of English and Some Implication for Teaching". Applied Linguistics 24/2: 223-242.

Reder, Anna (2006): Kollokationen in der Wortschatzarbeit. Wien: Praesens.

Reder, Anna (2015): „Überlegungen in Tendenzen in der Phraseodidaktik“. Deutschunterricht für Ungarn 27: 74-89.

Roth, Tobias (2014): Wortverbindungen und Verbindungen von Wörtern. Lexikographische und distributionelle Aspekte kombinatorischer Begriffe zwischen Syntax und Morphologie. Tübingen: Francke.

Siepmann, Dirk (2002): „Eigenschaften und Formen lexikalischer Kollokationen. Wider ein zu enges Verständnis“. Zeitschrift für französische Sprache und Literatur 112/3: 240-263. 
Siepmann, Dirk (2004): „Kollokationen und Fremdsprachenlernen. Imitation und Kreation, Figur und Hintergrund“. Praxis Fremdsprachenunterricht 2: 107-113.

Szulc, Aleksander (1971): „Linguistische, didaktische und methodische Probleme des Fremdsprachenunterrichts“. Deutsch als Fremdsprache 8/6: 328-337.

Szulc, Aleksander (1975): „Kognitivität und Habituation im Fremdsprachenunterricht“. In: Szulc, Aleksander (ed.): Sprachwissenschaft und Fremdsprachenunterricht. Poznań, Wydawnictwo Naukowe UAM: 25-37.

Szulc, Aleksander (1984): Podręczny słownik językoznawstwa stosowanego. Dydaktyka języków obcych. Warszawa: PWN.

Targońska, Joanna (2014): „Kollokationen - ein vernachlässigtes Gebiet der DaF Didaktik?““. Linguistik online 68/6: 127-149. doi: 10.13092/lo.68.1638.

Targońska, Joanna (2015a): „Welchen Kollokationsbegriff braucht die Fremdsprachendidaktik? Anregungen zu einer fremdsprachendidaktisch orientierten Auffassung des Kollokationsbegriffs“. Kwartalnik Neofilologiczny 62/3: 415-434. http://journals.pan.pl/dlibra/ publication/102875/edition/88889/content [10.03.2018].

Targońska, Joanna (2015b): „Kollokationen in DaF-Lehrwerken - eine empirische Studie zur Widerspiegelung der Kollokativität der Sprache in ausgewählten Lehrwerken“. Zielsprache Deutsch 42/3: 3-24.

Targońska, Joanna (2015c): „Theoretische Überlegungen zu Kollokationen in DaFLehrwerken“. Prace Językoznawcze. 17/3: 125-136. https://docs.google.com/viewer? url=http://uwm.edu.pl/polonistyka/pracejezykoznawcze/pliki/Prace-J-17-3-2015.pdf [10.03.2018].

Targońska, Joanna (2015d): „Konzeption der konventionellen Syntagmen von A. Szulc - ein bisher nicht beachteter Beitrag zur Kollokationsproblematik“. Lebende Sprachen 60/2: 360-377.

Targońska, Joanna (2016): „Zur Erfassung der Kollokationen in Vokabeltaschenbüchern und Vokabelheften für DaF-Lernende“. Prace Językoznawcze 18/4: 145-171. https://docs.go ogle.com/viewer?url=http://uwm.edu.pl/polonistyka/pracejezykoznawcze/pliki/PraceJez_18_4_2016.pdf [10.03.2018].

Wallner, Franziska (2014): Kollokationen in Wissenschaftssprachen. Zur lernerlexikographischen Relevanz ihrer wissenschaftssprachlichen Gebrauchsspezifika. Tübingen: Stauffenburg.

Wotjak, Barbara/Heine, Antje (2005): „Zur Abgrenzung und Beschreibung verbnominaler Wortverbindungen (Wortidiome, Funktionsverbgefüge, Kollokationen)“. Deutsch als Fremdsprache 3: 143-153. 